\title{
Initial terrestrial vertebrate diversity assessment in upland Cavite, Philippines
}

\author{
Ronaldo D. Lagat* and Rubie M. Causaren
}

\begin{abstract}
Cavite's remaining upland forest fragments are either remnants from commercial logging activities ca. 25-45 years ago or as a direct result of land conversions for agriculture or human settlements. These forest fragments are very significant because they represent areas where pockets of wildlife habitat still remain. The terrestrial vertebrates are often used to assess animal diversity because they are ideal biological indicators of environmental change and anthropogenic disturbances. The study aimed to determine terrestrial vertebrate diversity, conservation status, and identify major anthropogenic threats in these fragments. Terrestrial vertebrates were surveyed using a combination of strip-transect sampling, time-constrained searches, visual encounter survey (VES), and acoustic encounter survey (AES; for amphibians only), point counts, live trapping and mist netting from October 2014 to March 2016. Species richness and biodiversity estimation were computed using Shannon-Wiener Diversity Index, linear regression, detection and probability modeling using PAST, and confidence limits for nestedness (0.05 $\alpha$ ) using EpiTools. A total of 175 terrestrial vertebrates were documented and among the vertebrate groups, the birds had the highest observed diversity. Twenty-nine (19 birds, 3 mammals, 3 lizards, and 4 anurans) species are listed as threatened. Habitat loss and degradation due to the conversion of habitats to agricultural and/or residential areas remained to be the most prevalent threat in the remaining forested areas in upland Cavite. Baseline data generated shall be used in the different government biodiversity monitoring activities as the basis for impacts and mitigation and initial planning for the management and conservation of these remaining forest patches.
\end{abstract}

\section{KEYWORDS: amphibians, reptiles, mammals, birds, Luzon Island, modeling, anthropogenic threats}

\section{INTRODUCTION}

With more than 52,177 described species, the Philippines is regarded as one of the 17 megadiversity countries, which together contain $70-80 \%$ of global biodiversity (Mittermeier et al. 1997). The country houses approximately 38,000 animal species consisting of at least 35,000 invertebrates and at least 3,000 vertebrates (Ong et al. 2002). Of the 3,000+ vertebrates, $1121+$ terrestrial species (Ong et al. 2002; PBS 2014) include 107+ amphibians and 258+ reptiles (Alcala and Brown 1998; Diesmos and the Herpetofauna Working Group 2000; Brown et al. 2001; Brown pers. com.), 576+ birds

Biological Sciences Department \& Graduate Studies Department College of Science and Computer Studies

De La Salle University-Dasmariñas,

Dasmariñas City, Cavite, Philippines

*Corresponding email: rdlagat@dlsud.edu.ph

Date Submitted: 11 April 2018

Date Accepted: 30 April 2019
(Dickinson et al. 1991; Collar et al. 1994, 1999; WCSP 1997; Mallari and the Bird Working Group 2000; Mallari et al. 2001) and 179+ mammals (Heaney et al. 1998; Heaney and Mallari 2001). Of these $1121+$ species, $555+(\geq 50 \%)$ are endemic to the Philippines. These numbers represent rough estimates since many species are still undescribed (PBS 2014).

Apart from being a megadiversity country, the Philippines is also one of the 25 global biodiversity 'hotspots' (Myers et al. 2000) facing one of the highest levels of species endangerment. Habitat loss and fragmentation due to anthropogenic activities remain to be the gravest threats to general biodiversity loss (FAO, 2010; Jackson and Fahrig, 2013; Wu 2013). Worldwide, Southeast Asia has the highest relative rate of deforestation of any major tropical region (Woodcock et al., 2011; Rademaekers et al., 2010) and the Philippines has likely suffered the most devastating costs of large-scale deforestation (Tumaneng-Diete et al. $2005)$ in this region. Cavite ranked 73 rd in the country in terms of total forest cover with 1,864 ha $(1,852$ 
ha=broadleaf closed canopy + 12 ha=mangrove) and this represents only $1.49 \%$ total forest cover compared to its land area of 124,720 ha (Walpole, 2010: table 3).

The CALABARZON region is currently regarded as an "industrial belt of the country" (oxfordbusinessgroup.com) and Cavite (together with Batangas) now hosts the highest concentration of high-tech industries and electronics in the region. Historically, the province was not spared from logging and its remaining forest fragments (limited only to the upland areas) are either remnants from commercial logging activities ca. 25-45 years ago or as a direct result of land conversions for agriculture or human settlements (Liu et al. 1993; Tumaneng- Diete et al. 2005). These forest fragments are very significant because they represent areas where pockets of wildlife habitat still remain.

The terrestrial vertebrates are often used to assess animal diversity of terrestrial ecosystems because they are ideal biological indicators of environmental change and anthropogenic disturbances and are often used in predicting extinction (Yom-Tov and Geffen 2010; Chaudhary and Mooers 2018; Schmitt 2018) and planning conservation efforts (Jenkins et al. 2013). In addition, survey methods and taxa identification are less difficult compared to invertebrates due to availability of field guides and taxonomic keys (Siddig et al. 2015). Very few terrestrial vertebrate studies have been done in the province of Cavite and these were mostly limited to its protected area, Mt. Palay-Palay (including other peaksMt. Pico de Loro and Mt. Mataas-na-Gulod). Studies included those of fish (Jacinto and America 2005), amphibians (Celis et al. 1996; Paloma and Panganiban 1997; Maranan, 1999; Causaren 2009, 2012), reptiles (Lagat 1999, 2009, 2012), birds (Lorenzana and Rocamora 1997; Lalap and Ybanes 2006; Cuevas 2016), and mammals (Lardizabal and Maniago 1996; Lo and Quemuel 1998; Raroque and Valerio 1999; Lope and Hernandez 2008). Few studies also provided documentation on the landscape's faunal assemblage (Buenaventura et al. 2003, Guyamin 2004). Few studies on amphibians and reptiles have been conducted on some of the remaining secondary lowland forest fragments in Cavite (Causaren 2012, 2016; Lagat 2012; Causaren et al. 2016, 2017).

Some anthropogenic threats were observed in different forest fragments of Cavite and these included poaching, illegal logging, quarrying, charcoal-making, and 'kaingin' among others (Medecilo and Luyon 2006; Causaren 2012; Lagat 2012). Despite existing anthropogenic threats, the implementation of laws in relation to habitat and species protection is very weak thus continuously placing our native and endemic plant and animal species in grave peril. Given all these scenarios, the study aims to generate baseline information on the terrestrial vertebrate diversity status (focusing on four major terrestrial vertebrate taxa: amphibians, lizards, birds and mammals) in selected forested areas in upland mountainous areas in Cavite. Specifically, the study: 1) determines the terrestrial vertebrate species diversity of each forested area, 2) determines conservation status of these vertebrates, and 3) identifies current major threats to terrestrial vertebrate fauna in the study area. Baseline diversity information generated by this study would be useful to the Protected Area Management Board (PAMB) which is the managing and policy-making body of Mt. Palay-Palay. As representatives of De La Salle University-Dasmariñas, the research arm of PAMB in Cavite, we are tasked to submit an output as part of materializing an MOU between these units. Likewise, baseline data shall be submitted to the Municipal Environment and Natural Resources Officer (MENRO) of the different municipalities (where the forest fragments are situated) for biodiversity monitoring activities, as basis for impacts and mitigation and initial planning for the management and conservation of these remaining forest patches.

\section{STUdY AREA AND METHOdS}

Study sites. Five secondary lowland forest fragments in the province of Cavite (Figure 1, Table 1) were chosen as study sites. The forested fragments ranged in size from 6-640 ha and are situated at elevations $60-650$ masl. These forested fragments are either remnants of previous large-scale commercial logging ( 25 to 45 years ago) followed by land conversion for agriculture and massive urban development (Liu et al. 1993; Tumaneng-Diete et al. 2005). Site 1 (Amadeo) is a public area ca. 20 ha of secondary forest and is mainly riparian and partly reforested, at $272 \mathrm{~m}$ elevation, with coordinates: $14.2219^{\circ} \mathrm{N}, 120.9334^{\circ} \mathrm{E}$. The dominant trees are Dysoxylum gaudichianum and Pterospermum diversifolium. Site 2 in General Emilio Aguinaldo (or Bailen; henceforth GEA) is a public area and is also mainly riparian ca. 6 ha and at 60-70 m elevation, with coordinates: $14.2157^{\circ} \mathrm{N}, 120.7782^{\circ} \mathrm{E}$. It is dominated by Tarrenoidea wallichii, Kleinhovia hospita L. var. hospita and Chrysophyllum caimito (Medecilo and Lagat 2017). Site 3 (Indang) is a private area and mainly a riparian forested fragment ca. 10 ha and at $168 \mathrm{~m}$ elevation, with coordinates: $14.2238^{\circ} \mathrm{N}, 120.8507^{\circ}$ E. Being riparian, Indang's forested areas are dominated by Macaranga hispida, Ficus nota, M. multiglandulosa, M. tanarius, F. minahassae, F. septica, and Parkia roxburgii species which are either riparian species or those inhabiting other areas near water. Site 4 is Mt. Palay-Palay (only PA in Cavite) which is ca. 640 ha of secondary dipterocarp forest at $648 \mathrm{~m}$ elevation, with coordinates: $14.2394^{\circ} \mathrm{N}, 120.6531^{\circ} \mathrm{E}$. The remaining forest cover is estimated at $16 \%$ of its total land area (Environmental Science for Social Change, 2010) down 


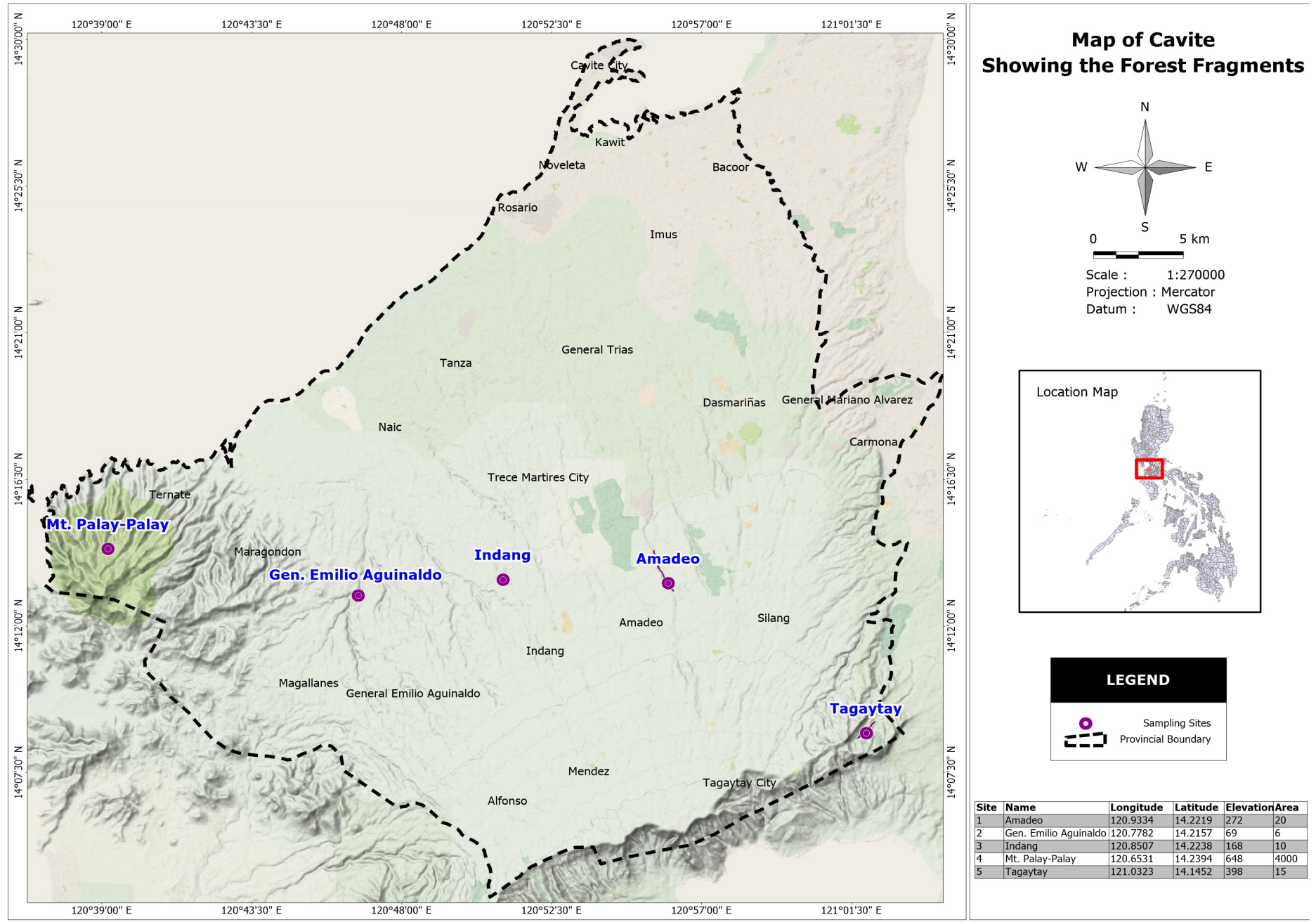

Figure 1. Map of Cavite delineated (black outline) from the neighboring provinces. The forest fragments are represented by purple dots. Modified from Google Earth 2017.

from $62.5 \%$ of land area about 20 years ago (DENR 1992). In terms of vegetation type, Mt. Palay-Palay can be classified as a lowland evergreen rain forest which includes the dipterocarp and mixed-dipterocarp forests (Causaren 2012; Lagat 2012; Medecilo and Lagat 2017). The vegetation of this fragment is similar to that of Mt. Makiling (Pancho, 1983). Though Planchonia spectabilis is the dominant species (relatively due to its larger basal area), Shorea guiso is the densest and most frequent species in natural forests. Site 5 (Tagaytay City) is a public area ca. 15 ha of riparian forest and at 60-70 $\mathrm{m}$ elevation, with coordinates: $14.1452^{\circ} \mathrm{N}, 121.0323^{\circ} \mathrm{E}$. It is dominated by Ficus minahassae, Alstonia scholaris, Leucaena leucocephala (Medecilo and Lagat 2017). Of the five forested fragments, four (except Mt. Palay-Palay) are mainly riparian forests which are usually located at very steep slopes making them somewhat inaccessible to human exploitation.

Sampling Regime. A total of eighteen $100 \mathrm{~m} \times 10 \mathrm{~m}$ transects for lizards, eighteen $100 \mathrm{~m} \times 10 \mathrm{~m}$ transects for amphibians, fourteen $100 \mathrm{~m} \times 10 \mathrm{~m}$ transects for large non-volant mammals; seven $100 \mathrm{~m} \times 10 \mathrm{~m}$ transects for small non-volant mammals and thirteen $2 \mathrm{~km}$ transects for birds was randomly established (Table 1). Field sampling for lizards and large non-volant mammals covered day and night visits (8:00am to $12: 00 \mathrm{nn}$ and $6: 00 \mathrm{pm}$ to $12: 00$ midnight) while for amphibians, bats and small non-volant mammals was during nighttime only (from $6: 00 \mathrm{pm}$ to $12: 00$ midnight). Bird sampling was conducted from 5:00 am until 10:00am. Field sampling was conducted from October 2014 to March 2016 by the same four persons with a total sampling effort of 72 hrs. for amphibians, $144 \mathrm{hrs}$. for lizards, $104 \mathrm{hrs}$. for birds, 112 hours for large non-volant mammals, 105 trap-nights for small non-volant mammals and 24 net nights for bats.

\section{Faunal Survey}

\section{A. Amphibian and lizard sampling}

A combination of strip-transect sampling, time-constrained searches, visual encounter survey (VES), and acoustic encounter survey (AES; for amphibians only) were used to determine species richness, abundance, and other ecological characteristics of different species (adopted and 


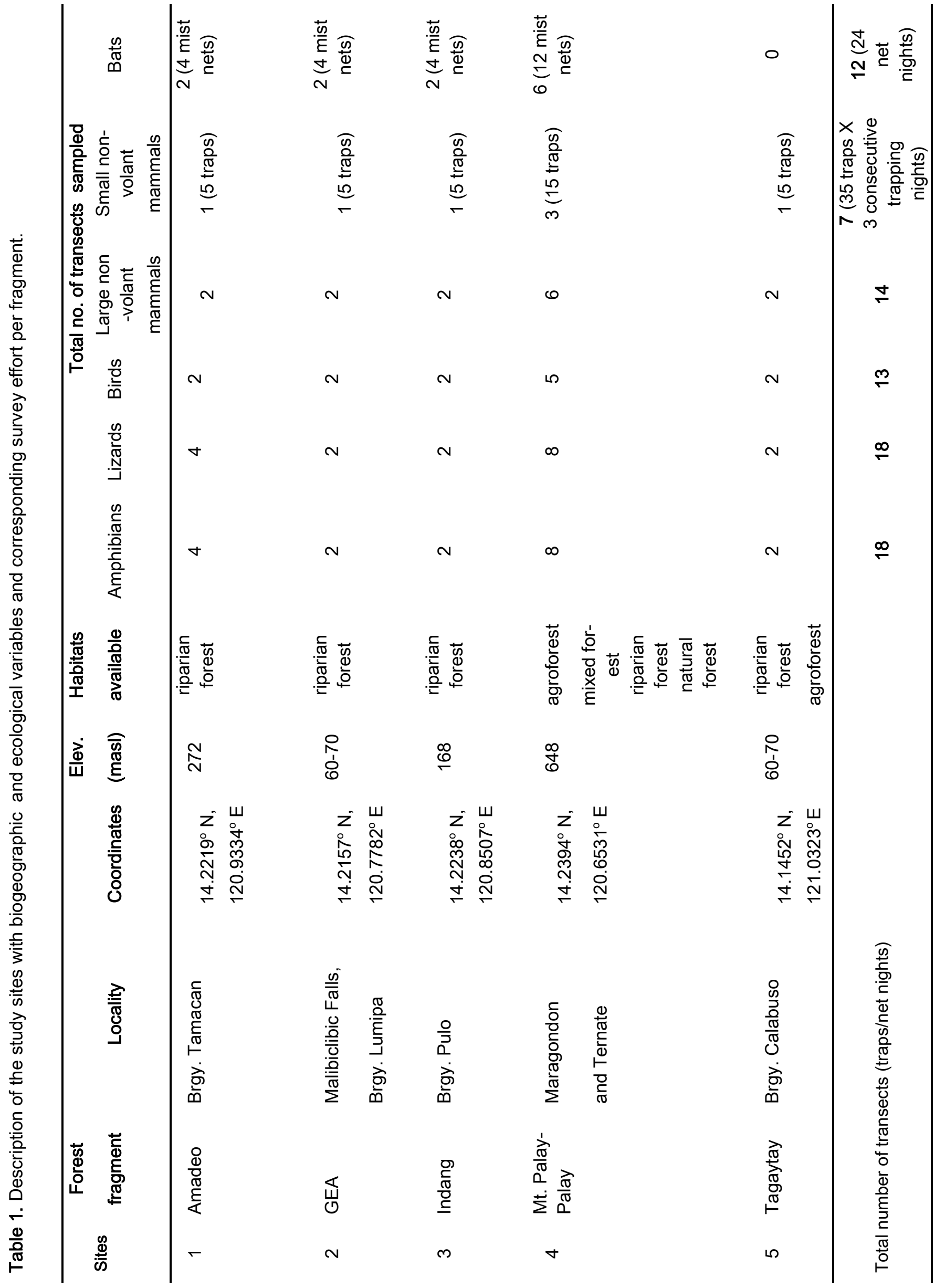


modified from Campbell and Christman 1982; Bury and Raphael 1983; Crump and Scott 1994; Heyer et al. 1994; Alcala et al., 2004; Diesmos et al., 2002; Diesmos, 2008;). Visual encounter surveys were conducted by walking through a transect (transect sampling) for a prescribed time of one hour (time-constrained searches), visually searching for animals. For amphibians, VES was supplemented with acoustic encounter survey where anuran species were identified by their calls (aural identification). The number of animals encountered (by both visual and aural methods) was recorded.

Strip transect and microhabitat sampling. A $100 \times 10$ m strip transect was marked at $10-\mathrm{m}$ intervals with numbered fluorescent flagging tapes that were labeled according to the transect number and point. For each transect, one hour was spent to sample all accessible microhabitats confined within. Microhabitats are specific areas within a community or habitat occupied by certain organisms because of micro-differences in moisture, light, and other conditions (availability of nutrients, protection from a predator, and the possibility of mating). Examples of microhabitats are forest litter, fallen logs, tree holes, rock crevices, spaces between buttresses of trees, forest shrubs, and axils of palms, epiphytes, tree ferns, aerial ferns and small trees. Prior to sampling, the first 2 points (points 0 and 1 ; with a distance of $10 \mathrm{~m}$ ) was marked and 6 minutes were spent in sampling this particular portion of the transect, after which all individuals whether seen, heard, or caught were recorded. Individuals that were caught were placed inside Ziploc $®$ plastic bags (individuals belonging to the same species were placed in one Ziploc $\circledast$ bag) and marked with the transect and point number. After sampling the first portion, the next point (point 2) was marked and another 6 minutes were spent to sample terrestrial vertebrates. This was done repeatedly until all the succeeding portions of the transect were sampled. This was done in order to minimize disturbance within the transect.

General collections were also carried out in all habitats and matrix habitats, but data were considered to note overall species diversity but not used in the computation of abundance. Data that were gathered and recorded included richness and abundance (individual counts [observed using both visual and aural surveys] and presence/absence data for every point in the transect), microhabitat, time of observation, and behavior of the animal during observation (e.g., calling, foraging, etc.).

\section{B. Birds}

Birds were sampled using the point counts or point transects (Bibby et al. 2000) which were used to provide estimates of the relative abundance of each bird species (Buckland et al. 2001). Five points were identified in a $2-\mathrm{km}$ line transect (400m apart). The total sampling time per transect was 120 minutes ( 3 to 5 minutes travel from one point to the next and 20 minutes observation time). This allowed the birds to settle for some time. Birds seen and heard within 20 minutes at each sampling spot were recorded.

\section{Mammals}

Ground mammals were surveyed using a combination of line transect (Plumptre and Reynolds 1994) and live trapping (O' Farrel et al. 1994) techniques. Line transect sampling followed the protocol for herps. In live trapping technique, a 100-meter transect was deployed with five equally designated spots where live traps were installed. Locally made steel-mesh traps $(28 \mathrm{~cm} \times 18 \mathrm{~cm} \times 12 \mathrm{~cm})$ were placed at or near ground level $(0-1 \mathrm{~m})$ within $20 \mathrm{~m}$ distance of designated spot along the $100-\mathrm{m}$ transect line with a total of five traps per transect. In each transect, trapping was conducted for three consecutive nights and traps were checked the morning after. A total of 105 trap-nights was employed throughout the study. For bats, two mist nets per transect (except in Tagaytay due to bad weather condition) were set at least $30 \mathrm{~m}$ apart at the nearest travel corridors. The nets were set after sunset and were kept open for 6 hours and checked every after 30 mins. Captured individuals were measured and identified to species level.

\section{Identification and Classification of Terrestrial Vertebrates}

Captured terrestrial vertebrates were measured, described, identified and were released at/near the sites of capture. Taxonomic identification and nomenclature of herps followed Alcala (1986), Brown et al. (1997a, b, c), Alcala and Brown (1998), Diesmos (1998, 1999, 2008), and Frost (2011); for birds, Wild Bird Club of the Philippines - Checklist of Birds of the Philippines 2018; and for mammals, Heaney et al. (2000, 2010) and Heaney and Rickart (2016). Selected voucher specimens were deposited in the Natural History Collection of De La Salle University- Dasmariñas, Cavite.

\section{Identification of Anthropogenic Threats}

Anthropogenic threats were observed and photo documented in different forested fragments in addition to results of interviews with the locals and DENR staff.

\section{Data Analysis}

Sampling sufficiency was checked based from the species effort curves generated using EstimateS 9.1.0 (Colwell 2016). Species richness and diversity estimation were represented by Shannon-Wiener Diversity Index ( $\mathrm{H}^{\prime}$ and $\mathrm{H}$ max) which were generated using Paleontological Statistics (PAST) Software Package for Education and Data Analysis (Hammer et al. 2001). Data from non-random searches/ overall collection were only considered to note overall species diversity but were not used in the computation of 
species richness and abundance. Estimates of Nestedness (which is a measure describing the distribution of observed species in all the sampling locations) and prevailing anthropogenic threats were used as bases for local conservation status of lizard and anuran species. Nestedness was expressed in terms of Proportion of Area Occupied (PAO) and confidence interval distribution at $0.05 \alpha$, generated using EpiTools (epidemiological online calculators). PAO makes use of the probability that the species is present and the sampling incidence that the species will be detected based on the observed detection history for a site over a series of survey occasion (MacKenzie et al. 2002, 2006). Species-area relationship was modeled using linear regression generated also from PAST.

\section{RESULTS AND DISCUSSION}

\section{Species diversity, endemism and distribution}

At least 175 species of amphibians, lizards, birds, and mammals are now known from upland Cavite, comprised of 15 species of frogs, 27 lizards, 12 mammals and 121 birds (Table 2). However, based from the species effort curves (Figure 2) the possibility of finding more species for birds and mammals is high since sampling was not very extensive (e.g., arboreal traps were not set for sampling other mammals) to
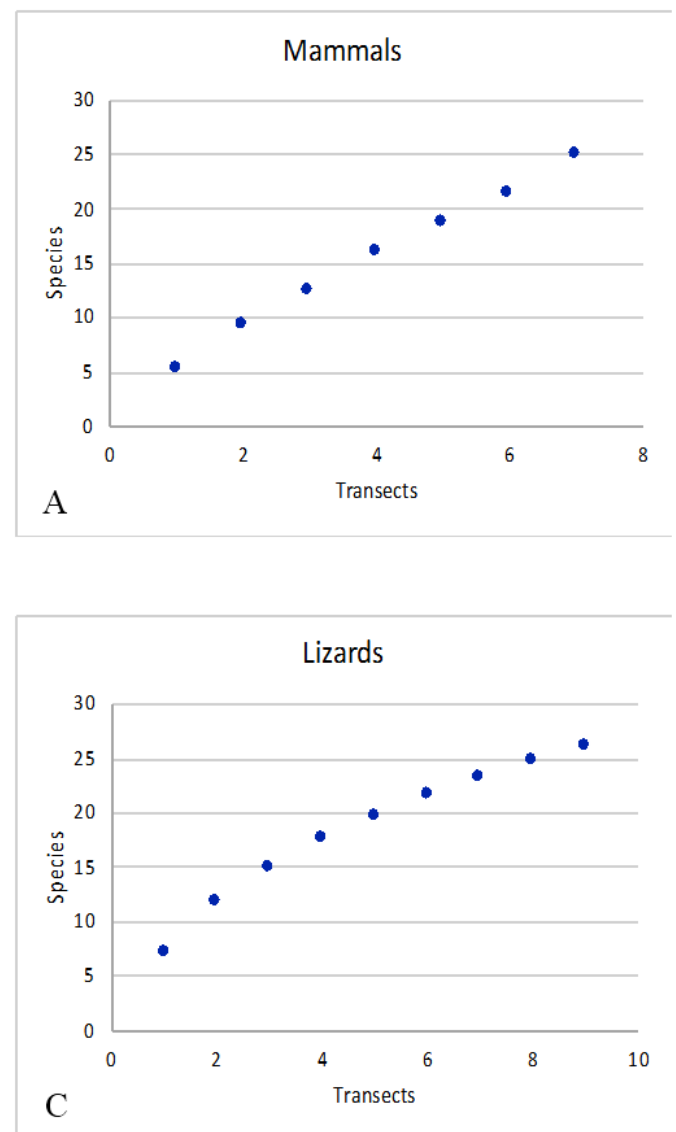

document all species. The most represented bird family was Columbidae with 14 species, mammal family was Pteropodidae with four species, lizard family was Scincidae with 17 species, and anuran families Ceratobatrachidae and Ranidae both with five species.

Considering current data, Mt. Palay-Palay registered the highest terrestrial vertebrate diversity for birds $(\mathrm{H}=4.12)$ and lizards $(\mathrm{H}=2.54)$, anuran diversity was observed highest in Amadeo $(\mathrm{H}=1.96)$ while GEA had the highest diversity in mammals $(\mathrm{H}=1.94)$ (Figure 3$)$. The most species-rich is Mt. Palay-Palay with $89.8 \%$ (157/175) species composition while the four fragments relatively had similar proportions ranging from $22-27 \%$ (Figure 4). The birds $(H=4.12)$ were observed to be the most diverse group in all the fragments sampled. Species endemicity was remarkably high with as many as 125 species (71\%; 10 mammals, 16 lizards, 9 anurans, 91 birds) endemic to the country. Data on the occurrence of each species per forested fragment and other pertinent information are summarized in Table 2.

Species-area distribution was observed to have a positive correlation (Figure 5), where the number of species increased with increasing area size (Preston 1960, 1962; Williams 1964; MacArthur and Wilson 1967; Pan 2013). This
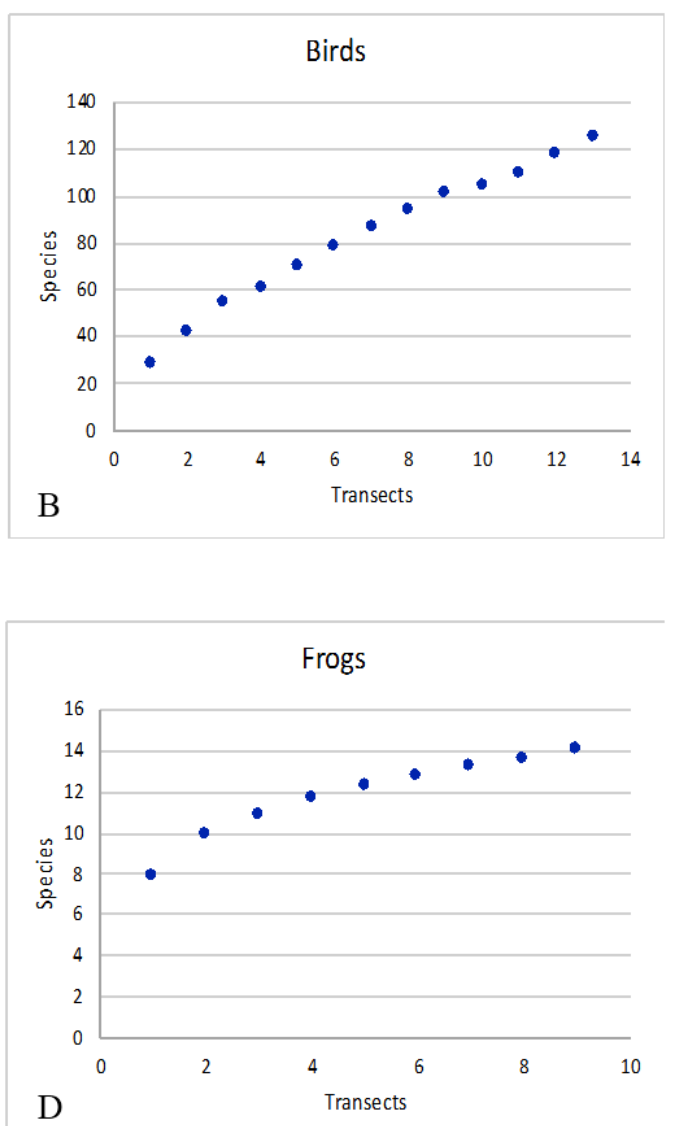

Figure 2. Sampling sufficiency graphs (generated using Estimate $S$ 9.1.0) for mammals (A), birds (B), lizards (C) and frogs (D). 
Table 2. Vertebrate fauna of upland Cavite. + indicates presence in forested fragments of $P=$ Mt. Palay-Palay, $I=I n d a n g, G=G e n e r a l$ Emilio Aguinaldo, $\mathrm{A}=$ Amadeo, and $\mathrm{T}=$ Tagaytay City. Ecological status, $E S(\mathrm{E}=$ Philippine endemic, $\mathrm{N}=\mathrm{Native}, \mathrm{W}=$ Widespread, $\mathrm{I}=$ Introduced) and Conservation status, CS (EN = Endangered, VU = Vulnerable, NT = Near Threatened, LC $=$ Least Concern, DD $=$ Data Deficient, LR = Lower Risk, NE = Not Evaluated) follow IUCN, 2017 (<www.iucnredlist.org>. Downloaded on 17 October 2017).

\begin{tabular}{|c|c|c|c|c|c|c|c|c|c|}
\hline Family & Taxa & English/Common name & $\mathbf{P}$ & I & G & A & $\mathrm{T}$ & ES & cs \\
\hline \multicolumn{10}{|l|}{ BIRDS } \\
\hline \multirow[t]{2}{*}{ Accipitridae } & $\begin{array}{l}\text { Haliastur indus (Boddaert, } \\
\text { 1783) }\end{array}$ & Brahminy Kite & + & & + & & & $\mathrm{N}$ & LC \\
\hline & $\begin{array}{l}\text { Spilornis holospilus } \\
\text { (Vigors, 1831) }\end{array}$ & Philippine Serpent-Eagle & + & & & & & $E$ & LC \\
\hline \multirow[t]{5}{*}{ Alcedinidae } & $\begin{array}{l}\text { Actenoides lindsayi } \\
\text { (Vigors, 1831) }\end{array}$ & Spotted Kingfisher & + & & & + & + & $\mathrm{E}$ & LC \\
\hline & $\begin{array}{l}\text { Ceyx cyanopectus } \\
\text { (Lafresnaye, 1840) }\end{array}$ & Indigo-banded Kingfisher & & + & & & & $E$ & LC \\
\hline & $\begin{array}{l}\text { Ceyx melanurus } \\
\text { (Kaup, 1848) }\end{array}$ & Philippine Dwarf-kingfisher & + & & & & & $E$ & VU \\
\hline & $\begin{array}{l}\text { Halcyon gularis } \\
\text { (Kuhl, 1820) }\end{array}$ & White-throated Kingfisher & + & & & + & + & W & LC \\
\hline & $\begin{array}{l}\text { Todiramphus chloris } \\
\text { (Boddaert, 1783) }\end{array}$ & White-collared Kingfisher & + & + & + & + & + & W & LC \\
\hline Anatidae & $\begin{array}{l}\text { Anas luzonica Fraser, } \\
1839\end{array}$ & Philippine Duck & + & & + & & & $E$ & VU \\
\hline \multirow[t]{3}{*}{ Apodidae } & $\begin{array}{l}\text { Collocalia esculenta } \\
\text { isonota Oberholser, } 1906\end{array}$ & Glossy Swiftlet & + & + & + & + & + & $E$ & LC \\
\hline & $\begin{array}{l}\text { Collocalia troglodytes } \\
\text { Gray, GR, } 1845\end{array}$ & Pygmy Swiftlet & & & + & & & $E$ & LC \\
\hline & $\begin{array}{l}\text { Mearnsia picina } \\
\text { (Tweeddale, 1879) }\end{array}$ & $\begin{array}{l}\text { Philippine Spine-tailed } \\
\text { Swift }\end{array}$ & + & & & & & $E$ & NT \\
\hline \multirow[t]{5}{*}{ Ardeidae } & $\begin{array}{l}\text { Ardea alba } \\
\text { Linnaeus, } 1758\end{array}$ & Great Egret & + & & & & & W & LC \\
\hline & $\begin{array}{l}\text { Ardeola bacchus } \\
\text { (Bonaparte, 1855) }\end{array}$ & Chinese Pond-Heron & + & & & & & $\mathrm{N}$ & LC \\
\hline & $\begin{array}{l}\text { Bubulcus coromandus } \\
\text { (Boddaert, 1783) }\end{array}$ & Cattle Egret & & + & & & & W & LC \\
\hline & $\begin{array}{l}\text { Egretta garzetta nigripes } \\
\text { (Temminck, 1840) }\end{array}$ & Eastern Reef-Egret & + & & & & & W & LC \\
\hline & $\begin{array}{l}\text { Nycticorax caledonicus } \\
\text { (Gmelin, JF, 1789) }\end{array}$ & Rufous Night-heron & + & & & & & W & LC \\
\hline Artamidae & $\begin{array}{l}\text { Artamus leucorynchus } \\
\text { (Linnaeus, 1771) }\end{array}$ & $\begin{array}{l}\text { White-breasted } \\
\text { Woodswallow }\end{array}$ & + & & & & & W & LC \\
\hline \multirow[t]{2}{*}{ Bucerotidae } & $\begin{array}{l}\text { Buceros hydrocorax } \\
\text { Linnaeus, } 1766\end{array}$ & Rufous Hornbill & + & & & & & $E$ & VU \\
\hline & $\begin{array}{l}\text { Penelopides manillae } \\
\text { (Boddaert, 1783) }\end{array}$ & Luzon Hornbill & + & & & & & $E$ & LC \\
\hline
\end{tabular}


Philippine Journal of Systematic Biology | Lagat,R.D. \& Causaren,R.M.: Terrestrial vertebrate diversity assessment in upland Cavite

\begin{tabular}{|c|c|c|c|c|c|c|c|c|c|}
\hline Family & Taxa & English/Common name & $\mathbf{P}$ & I & G & A & $\mathrm{T}$ & ES & CS \\
\hline \multirow[t]{6}{*}{ Campephagidae } & $\begin{array}{l}\text { Coracina striata } \\
\text { (Boddaert, 1783) }\end{array}$ & Bar-bellied Cuckoo-shrike & + & & & & & W & $\mathrm{LC}$ \\
\hline & $\begin{array}{l}\text { Coracina coerulescens } \\
\text { (Blyth, 1842) }\end{array}$ & Blackish Cuckooshrike & + & & & & & $\mathrm{E}$ & LC \\
\hline & $\begin{array}{l}\text { Coracina mindanensis } \\
\text { (Tweeddale, 1879) }\end{array}$ & $\begin{array}{l}\text { Black-bibbed Cuckoo- } \\
\text { shrike }\end{array}$ & + & & & & & $\mathrm{E}$ & VU \\
\hline & $\begin{array}{l}\text { Lalage melanoleuca } \\
\text { melanoleuca (Blyth, 1861) }\end{array}$ & $\begin{array}{l}\text { Northern Black-and-white } \\
\text { Triller }\end{array}$ & + & & & & & $\mathrm{E}$ & $\mathrm{LC}$ \\
\hline & $\begin{array}{l}\text { Lalage nigra nigra } \\
\text { (Forster, JR, 1781) }\end{array}$ & Pied Triller & + & & + & & & W & $\mathrm{LC}$ \\
\hline & $\begin{array}{l}\text { Pericrocotus speciosus } \\
\text { (Latham, 1790) }\end{array}$ & Scarlet Minivet & + & & & & & $\mathrm{E}$ & NE \\
\hline Caprimulgidae & $\begin{array}{l}\text { Lyncornis macrotis } \\
\text { (Vigors, 1831) }\end{array}$ & Great Eared-Nightjar & + & & & & & $\mathrm{E}$ & $\mathrm{LC}$ \\
\hline \multirow[t]{3}{*}{ Cisticolidae } & $\begin{array}{l}\text { Cisticola juncidis } \\
\text { (Rafinesque, 1810) }\end{array}$ & Zitting Cisticola & + & & & & & W & $\mathrm{LC}$ \\
\hline & $\begin{array}{l}\text { Orthotomus castaneiceps } \\
\text { Walden, } 1872\end{array}$ & Philippine Tailorbird & + & & & + & + & $\mathrm{E}$ & LC \\
\hline & $\begin{array}{l}\text { Chalcophaps indica indica } \\
\text { (Linnaeus, 1758) }\end{array}$ & Grey-capped Emerald & + & & & & & $N$ & $\mathrm{LC}$ \\
\hline \multirow[t]{11}{*}{ Columbidae } & $\begin{array}{l}\text { Columba livia Gmelin, } \\
\text { JF, } 1789\end{array}$ & $\begin{array}{l}\text { Rock Dove, Common } \\
\text { Pigeon, Rock Dove, Rock } \\
\text { Dovel }\end{array}$ & & + & + & + & + & $\mathrm{E}$ & $\mathrm{LC}$ \\
\hline & $\begin{array}{l}\text { Ducula aenea (Linnaeus, } \\
\text { 1766) }\end{array}$ & Green Imperial-Pigeon & + & & & & & $\mathrm{E}$ & $\mathrm{LC}$ \\
\hline & $\begin{array}{l}\text { Ducula carola (Bonaparte, } \\
\text { 1854) }\end{array}$ & Spotted Imperial-Pigeon & + & & & & & $\mathrm{E}$ & VU \\
\hline & $\begin{array}{l}\text { Ducula poliocephala } \\
\text { (Gray, GR, 1844) }\end{array}$ & $\begin{array}{l}\text { Pink-bellied Imperial- } \\
\text { Pigeon }\end{array}$ & + & & & & & $\mathrm{E}$ & NT \\
\hline & $\begin{array}{l}\text { Gallicolumba luzonica } \\
\text { Iuzonica (Scopoli, 1786) }\end{array}$ & $\begin{array}{l}\text { Luzon Bleeding-heart, } \\
\text { Bleeding Heart Dove, } \\
\text { Bleeding Heart Pigeon }\end{array}$ & + & & & & & $\mathrm{E}$ & NT \\
\hline & $\begin{array}{l}\text { Geopelia striata } \\
\text { (Linnaeus, 1766) }\end{array}$ & $\begin{array}{l}\text { Zebra Dove, Barred } \\
\text { Ground Dove, Peaceful } \\
\text { Dove }\end{array}$ & + & & & + & + & 1 & $\mathrm{LC}$ \\
\hline & $\begin{array}{l}\text { Macropygia tenuirostris } \\
\text { Bonaparte, } 1854\end{array}$ & Philippine Cuckoo-Dove & + & & & & & $\mathrm{E}$ & $\mathrm{LC}$ \\
\hline & $\begin{array}{l}\text { Phapitreron leucotis } \\
\text { leucotis (Temminck, } \\
\text { 1823) }\end{array}$ & White-eared Brown-dove & + & + & + & + & + & $\mathrm{E}$ & $\mathrm{LC}$ \\
\hline & $\begin{array}{l}\text { Ptilinopus leclancheri } \\
\text { (Bonaparte, 1855) }\end{array}$ & Black-chinned Fruit-Dove & + & & & & & $\mathrm{E}$ & LC \\
\hline & $\begin{array}{l}\text { Ptilinopus merrilli } \\
\text { (McGregor, 1916) }\end{array}$ & $\begin{array}{l}\text { Cream-bellied Fruit-Dove, } \\
\text { Cream-breasted Fruit Dove }\end{array}$ & + & & & & & $E$ & NT \\
\hline & $\begin{array}{l}\text { Ptilinopus occipitalis Gray, } \\
\text { GR, } 1844\end{array}$ & Yellow-breasted Fruit-Dove & + & & & & & E & $\mathrm{LC}$ \\
\hline
\end{tabular}




\begin{tabular}{|c|c|c|c|c|c|c|c|c|c|}
\hline Family & Taxa & English/Common name & $\mathbf{P}$ & I & G & A & $\mathrm{T}$ & ES & CS \\
\hline \multirow[t]{2}{*}{ Columbidae } & $\begin{array}{l}\text { Streptopelia chinensis } \\
\text { tigrina (Temminck, 1809) }\end{array}$ & Spotted Dove & + & & & & & 1 & NE \\
\hline & $\begin{array}{l}\text { Treron axillaris axillaris } \\
\text { (Gmelin, JF, 1789) }\end{array}$ & Philippine Green-pigeon & + & & & & & $E$ & LC \\
\hline \multirow[t]{2}{*}{ Corvidae } & $\begin{array}{l}\text { Corvus enca } \\
\text { (Horsfield, 1821) }\end{array}$ & Slender-billed Crow & + & + & + & & & $E$ & LC \\
\hline & $\begin{array}{l}\text { Corvus philippinus } \\
\text { (Bonaparte, 1853) }\end{array}$ & Philippines Crow & + & + & & + & + & $E$ & NE \\
\hline \multirow[t]{6}{*}{ Cuculidae } & $\begin{array}{l}\text { Centropus sinensis } \\
\text { bubutus Horsfield, } 1821\end{array}$ & Greater Coucal & + & & + & & & $\mathrm{N}$ & LC \\
\hline & $\begin{array}{l}\text { Centropus viridis } \\
\text { (Scopoli, 1786) }\end{array}$ & Philippine Coucal & + & & & & & $E$ & LC \\
\hline & $\begin{array}{l}\text { Dasylophus superciliosus } \\
\text { (Dumont, 1823) }\end{array}$ & Red-crested Malkoha & + & + & & & & $E$ & LC \\
\hline & $\begin{array}{l}\text { Eudynamys scolopaceus } \\
\text { (Linnaeus, 1758) }\end{array}$ & Western Koel & + & & & & & $E$ & LC \\
\hline & $\begin{array}{l}\text { Lepidogrammus cumingi } \\
\text { (Fraser, 1839) }\end{array}$ & Scale-feathered Malkoha & + & & & & & $E$ & LC \\
\hline & $\begin{array}{l}\text { Surniculus velutinus } \\
\text { chalybaeus } \\
\text { Salomonsen, } 1953\end{array}$ & Philippine Drongo-Cuckoo & + & & & & & $E$ & LC \\
\hline \multirow[t]{6}{*}{ Dicaeidae } & $\begin{array}{l}\text { Dicaeum australe } \\
\text { (Hermann, 1783) }\end{array}$ & $\begin{array}{l}\text { Red-keeled Flowerpecker, } \\
\text { Red-striped Flowerpecker }\end{array}$ & + & + & & & & $E$ & LC \\
\hline & $\begin{array}{l}\text { Dicaeum bicolor } \\
\text { inexpectatum } \\
\text { (Hartert, E, 1895) }\end{array}$ & Bicolored Flowerpecker & + & & & & & $E$ & LC \\
\hline & $\begin{array}{l}\text { Dicaeum hypoleucum } \\
\text { obscurum } \\
\text { Ogilvie-Grant, } 1894\end{array}$ & Buzzing Flowerpecker & + & & & & & $E$ & LC \\
\hline & Dicaeum pygmaeum & & & & & & & & \\
\hline & $\begin{array}{l}\text { pygmaeum } \\
\text { (von Kittlitz, 1833) }\end{array}$ & Pygmy Flowerpecker & + & & & & & $E$ & LC \\
\hline & $\begin{array}{l}\text { Prionochilus olivaceus } \\
\text { samarensis Steere, } 1890\end{array}$ & Olive-backed Flowerpecker & + & & & & & $E$ & LC \\
\hline Dicruridae & $\begin{array}{l}\text { Dicrurus balicassius } \\
\text { balicassius } \\
\text { (Linnaeus, 1766) }\end{array}$ & Balicassiao & + & + & + & + & + & $E$ & LC \\
\hline \multirow[t]{2}{*}{ Estrildidae } & $\begin{array}{l}\text { Lonchura atricapilla jagori } \\
\text { (von Martens, CE, 1866) }\end{array}$ & Chestnut Munia & & & & + & + & 1 & LC \\
\hline & $\begin{array}{l}\text { Lonchura leucogastra } \\
\text { (Blyth, 1846) }\end{array}$ & White-bellied Munia & & + & & + & + & $E$ & LC \\
\hline Falconidae & $\begin{array}{l}\text { Microhierax erythrogenys } \\
\text { (Vigors, 1831) }\end{array}$ & Philippine Falconet & + & & & & & $E$ & LC \\
\hline
\end{tabular}




\begin{tabular}{|c|c|c|c|c|c|c|c|c|c|}
\hline Family & Taxa & English/Common name & $\mathbf{P}$ & $\mathrm{I}$ & G & A & $\mathbf{T}$ & ES & CS \\
\hline \multirow[t]{2}{*}{ Hirundinidae } & $\begin{array}{l}\text { Hirundo rustica } \\
\text { Linnaeus, } 1758\end{array}$ & $\begin{array}{l}\text { Barn Swallow, European } \\
\text { Swallow, Swallow }\end{array}$ & + & & & & & $\mathrm{N}$ & LC \\
\hline & $\begin{array}{l}\text { Hirundo tahitica javanica } \\
\text { Sparrman, } 1789\end{array}$ & Tahiti Swallow & + & & & & & 1 & LC \\
\hline Irenidae & $\begin{array}{l}\text { Irena cyanogastra } \\
\text { Vigors, } 1831\end{array}$ & Philippine Fairy-bluebird & + & & & & & $E$ & NT \\
\hline \multirow[t]{3}{*}{ Laniidae } & $\begin{array}{l}\text { Lanius cristatus } \\
\text { lucionensis } \\
\text { Linnaeus, } 1766\end{array}$ & Brown Shrike & + & + & + & + & + & $\mathrm{N}$ & LC \\
\hline & $\begin{array}{l}\text { Lanius schach nasutus } \\
\text { Scopoli, } 1786\end{array}$ & Long-tailed Shrike & + & & & & & $\mathrm{N}$ & LC \\
\hline & $\begin{array}{l}\text { Lanius validirostris } \\
\text { Ogilvie-Grant, } 1894\end{array}$ & Mountain Shrike & + & & & & & $E$ & NT \\
\hline Locustellidae & $\begin{array}{l}\text { Megalurus palustris } \\
\text { forbesi Bangs, } 1919\end{array}$ & Striated Grassbird & + & & & & & $E$ & LC \\
\hline Megalaimidae & $\begin{array}{l}\text { Psilopogon } \\
\text { haemacephalus } \\
\text { haemacephalus } \\
\text { (Statius Muller, 1776) }\end{array}$ & Coppersmith Barbet & + & + & & + & + & $E$ & LC \\
\hline \multirow[t]{2}{*}{ Meropidae } & $\begin{array}{l}\text { Merops americanus } \\
\text { Linnaeus, } 1758\end{array}$ & Rufous-crowned Bee-eater & + & & & & & $E$ & LC \\
\hline & $\begin{array}{l}\text { Merops philippinus } \\
\text { Linnaeus, } 1767\end{array}$ & Blue-tailed Bee-eater & + & & & & & $E$ & LC \\
\hline \multirow[t]{4}{*}{ Monarchidae } & $\begin{array}{l}\text { Hypothymis azurea } \\
\text { azurea (Boddaert, 1783) }\end{array}$ & Black-naped Monarch & + & & & & & $E$ & LC \\
\hline & $\begin{array}{l}\text { Hypothymis helenae } \\
\text { (Steere, 1890) }\end{array}$ & $\begin{array}{l}\text { Short-crested Monarch, } \\
\text { Short-crested Blue } \\
\text { Monarch }\end{array}$ & & & & + & + & $E$ & NT \\
\hline & $\begin{array}{l}\text { Terpsiphone cinnamomea } \\
\text { unirufa Salomonsen, } 1937\end{array}$ & $\begin{array}{l}\text { Southern Rufous } \\
\text { Paradise-Flycatcher }\end{array}$ & + & & & & & $E$ & LC \\
\hline & $\begin{array}{l}\text { Terpsiphone cyanescens } \\
\text { (Sharpe, 1877) }\end{array}$ & Blue Paradise-Flycatcher & & + & & + & + & $E$ & LC \\
\hline \multirow[t]{3}{*}{ Motacillidae } & $\begin{array}{l}\text { Anthus gustavi gustavi } \\
\text { Swinhoe, } 1863\end{array}$ & Pechora Pipit & + & & & & & $\mathrm{N}$ & LC \\
\hline & $\begin{array}{l}\text { Dendronanthus indicus } \\
\text { (Gmelin, JF, 1789) }\end{array}$ & Forest Wagtail & + & + & & & & 1 & LC \\
\hline & $\begin{array}{l}\text { Motacilla cinerea } \\
\text { Tunstall, } 1771\end{array}$ & Grey Wagtail, Gray Wagtail & + & & & & & $\mathrm{N}$ & LC \\
\hline \multirow[t]{3}{*}{ Muscicapidae } & $\begin{array}{l}\text { Copsychus mindanensis } \\
\text { (Boddaert, 1783) }\end{array}$ & Philippine Magpie-Robin & + & & & + & + & $E$ & LC \\
\hline & $\begin{array}{l}\text { Cyornis herioti } \\
\text { Wardlaw Ramsay, } 1886\end{array}$ & $\begin{array}{l}\text { Blue-breasted } \\
\text { Blue-Flycatcher }\end{array}$ & + & & + & & & $E$ & NT \\
\hline & $\begin{array}{l}\text { Cyornis rufigastra blythi } \\
\text { (Giebel, 1875) }\end{array}$ & Mangrove Blue-Flycatcher & + & & + & & & $E$ & LC \\
\hline
\end{tabular}




\begin{tabular}{|c|c|c|c|c|c|c|c|c|c|}
\hline Family & Taxa & English/Common name & $\mathbf{P}$ & $\mathrm{I}$ & G & A & $\mathrm{T}$ & ES & $\mathrm{CS}$ \\
\hline \multirow[t]{5}{*}{ Muscicapidae } & $\begin{array}{l}\text { Eumyias panayensis } \\
\text { Sharpe, } 1877\end{array}$ & $\begin{array}{l}\text { Turquoise Flycatcher, } \\
\text { Island Flycatcher }\end{array}$ & + & & & & & $E$ & LC \\
\hline & $\begin{array}{l}\text { Ficedula Iuzoniensis } \\
\text { (Ogilvie-Grant, 1894) }\end{array}$ & Thicket Flycatcher & + & & & & & $E$ & LC \\
\hline & $\begin{array}{l}\text { Copsychus luzoniensis } \\
\text { (Kittlitz, 1832) }\end{array}$ & White-browed Shama & + & & & & & $E$ & LC \\
\hline & $\begin{array}{l}\text { Monticola solitarius } \\
\text { philippensis (Statius } \\
\text { Muller, 1776) }\end{array}$ & $\begin{array}{l}\text { Blue Rock-thrush, Blue } \\
\text { Rock Thrush, Blue Rock- } \\
\text { Thrush }\end{array}$ & + & & & & & $\mathrm{E}$ & LC \\
\hline & $\begin{array}{l}\text { Saxicola caprata caprata } \\
\text { (Linnaeus, 1766) }\end{array}$ & $\begin{array}{l}\text { Pied Bushchat, Pied Bush } \\
\text { Chat, Pied Stonechat }\end{array}$ & + & & & + & + & $\mathrm{N}$ & LC \\
\hline \multirow[t]{6}{*}{ Nectariniidae } & $\begin{array}{l}\text { Aethopyga flagrans } \\
\text { Oustalet, } 1876\end{array}$ & Flaming Sunbird & + & & & & & $E$ & LC \\
\hline & $\begin{array}{l}\text { Anthreptes griseigularis } \\
\text { birgitae } \\
\text { Salomonsen, } 1953\end{array}$ & Grey-throated Sunbird & + & & & + & + & $E$ & LC \\
\hline & $\begin{array}{l}\text { Anthreptes malacensis } \\
\text { (Scopoli, 1786) }\end{array}$ & Brown-throated Sunbird & + & & & + & + & $E$ & LC \\
\hline & $\begin{array}{l}\text { Arachnothera clarae } \\
\text { luzonensis } \\
\text { Alcasid \& Gonzales, } 1968\end{array}$ & Naked-faced Spider Hunter & + & + & + & + & + & $\mathrm{E}$ & LC \\
\hline & $\begin{array}{l}\text { Cinnyris jugularis jugularis } \\
\text { (Linnaeus, 1766) }\end{array}$ & Olive-backed Sunbird & + & & & & & $E$ & LC \\
\hline & $\begin{array}{l}\text { Leptocoma sperata } \\
\text { (Linnaeus, 1766) }\end{array}$ & Purple-throated Sunbird & + & & & & & $E$ & LC \\
\hline Oriolidae & $\begin{array}{l}\text { Oriolus chinensis } \\
\text { Linnaeus, } 1766\end{array}$ & Black-naped Oriole & + & & + & + & + & $E$ & LC \\
\hline Paridae & $\begin{array}{l}\text { Pardaliparus elegans } \\
\text { Lesson, } 1831\end{array}$ & Elegant Tit & + & + & & + & + & $E$ & LC \\
\hline Passeridae & $\begin{array}{l}\text { Passer montanus } \\
\text { (Linnaeus, 1758) }\end{array}$ & $\begin{array}{l}\text { Eurasian Tree Sparrow, } \\
\text { Tree Sparrow }\end{array}$ & + & + & + & + & + & 1 & $\mathrm{LC}$ \\
\hline \multirow[t]{2}{*}{ Phasianidae } & $\begin{array}{l}\text { Francolinus pintadeanus } \\
\text { (Scopoli, 1786) }\end{array}$ & Chinese Francolin & + & & & & & 1 & LC \\
\hline & $\begin{array}{l}\text { Synoicus chinensis } \\
\text { lineatus (Scopoli, 1786) }\end{array}$ & $\begin{array}{l}\text { Asian Blue Quail, Blue- } \\
\text { breasted Quail, Blue Quail, } \\
\text { King Quail }\end{array}$ & & & + & & & $\mathrm{N}$ & LC \\
\hline \multirow[t]{5}{*}{ Picidae } & Chrysocolaptes & & & & & & & & \\
\hline & $\begin{array}{l}\text { haematribon (Wagler, } \\
\text { 1827) }\end{array}$ & Luzon Flameback & + & & & & & $E$ & $\mathrm{LC}$ \\
\hline & $\begin{array}{l}\text { Dendrocopos maculatus } \\
\text { validirostris (Blyth, 1849) }\end{array}$ & $\begin{array}{l}\text { Philippine Pygmy } \\
\text { Woodpecker }\end{array}$ & + & & & & & $E$ & $\mathrm{LC}$ \\
\hline & $\begin{array}{l}\text { Mulleripicus funebris } \\
\text { (Valenciennes, 1826) }\end{array}$ & $\begin{array}{l}\text { Northern Sooty } \\
\text { Woodpecker }\end{array}$ & + & & & & & $E$ & NT \\
\hline & $\begin{array}{l}\text { Mulleripicus pulverulentus } \\
\text { (Temminck, 1826) }\end{array}$ & Great Slaty Woodpecker & + & & & & & $E$ & VU \\
\hline
\end{tabular}




\begin{tabular}{|c|c|c|c|c|c|c|c|c|c|}
\hline Family & Taxa & English/Common name & $\mathbf{P}$ & I & G & A & $\mathbf{T}$ & ES & CS \\
\hline Pittidae & $\begin{array}{l}\text { Erythropitta erythrogaster } \\
\text { Temminck, } 1823\end{array}$ & Philippine Pitta & + & & & & & $E$ & LC \\
\hline Podargidae & $\begin{array}{l}\text { Batrachostomus septimus } \\
\text { Tweeddale, } 1877\end{array}$ & Philippine Frogmouth & + & & & & & $E$ & LC \\
\hline \multirow[t]{2}{*}{ Psittacidae } & $\begin{array}{l}\text { Bolbopsittacus lunulatus } \\
\text { (Scopoli, 1786) }\end{array}$ & Guaiabero & + & & & & & $\mathrm{E}$ & LC \\
\hline & $\begin{array}{l}\text { Loriculus philippensis } \\
\text { philippensis (Statius } \\
\text { Muller, 1776) }\end{array}$ & $\begin{array}{l}\text { Philippine Hanging-parrot, } \\
\text { Colasisi, Philippine } \\
\text { Hanging Parrot }\end{array}$ & + & & & & & $E$ & $\mathrm{LC}$ \\
\hline \multirow[t]{2}{*}{ Pycnonotidae } & $\begin{array}{l}\text { Hypsipetes philippinus } \\
\text { philippinus } \\
\text { (Forster, JR, 1795) }\end{array}$ & Philippine Bulbul & + & + & + & + & + & $E$ & LC \\
\hline & $\begin{array}{l}\text { Pycnonotus goiavier } \\
\text { goiavier (Scopoli, 1786) }\end{array}$ & Yellow-vented Bulbul & + & & & & & $E$ & $\mathrm{LC}$ \\
\hline \multirow[t]{3}{*}{ Rallidae } & $\begin{array}{l}\text { Gallus gallus } \\
\text { (Linnaeus, } 1758 \text { ) }\end{array}$ & Red Junglefowl & + & & + & & & $\mathrm{N}$ & LC \\
\hline & $\begin{array}{l}\text { Hypotaenidia torquata } \\
\text { torquata (Linnaeus, 1766) }\end{array}$ & Barred Rail & + & & & + & + & $\mathrm{E}$ & $\mathrm{LC}$ \\
\hline & $\begin{array}{l}\text { Rallina fasciata } \\
\text { (Raffles, 1822) }\end{array}$ & Red-legged Crake & + & & & & & $\mathrm{N}$ & $\mathrm{LC}$ \\
\hline \multirow[t]{2}{*}{ Rhipiduridae } & $\begin{array}{l}\text { Rhipidura cyaniceps } \\
\text { cyaniceps (Cassin, 1855) }\end{array}$ & Blue-headed Fantail & + & & & & & $E$ & $\mathrm{LC}$ \\
\hline & $\begin{array}{l}\text { Rhipidura nigritorquis } \\
\text { (Sparrman, 1788) }\end{array}$ & Philippine Pied Fantail & + & + & + & & & $E$ & LC \\
\hline Scotocercidae & $\begin{array}{l}\text { Phyllergates cucullatus } \\
\text { philippinus } \\
\text { (Hartert, E, 1897) }\end{array}$ & Mountain Tailorbird & & & & & & $\mathrm{E}$ & $\mathrm{LC}$ \\
\hline \multirow[t]{3}{*}{ Strigidae } & $\begin{array}{l}\text { Bubo philippensis } \\
\text { (Kaup, 1851) }\end{array}$ & Philippine Eagle-owl, & + & & & & & $E$ & VU \\
\hline & $\begin{array}{l}\text { Otus longicornis } \\
\text { (Ogilvie-Grant, 1894) }\end{array}$ & $\begin{array}{l}\text { Luzon Highland Scops-owl, } \\
\text { Luzon Scops-Owl }\end{array}$ & + & & & & & $\mathrm{E}$ & NT \\
\hline & $\begin{array}{l}\text { Otus megalotis } \\
\text { (Walden, 1875) }\end{array}$ & $\begin{array}{l}\text { Luzon Lowland Scops-owl, } \\
\text { Philippine Scops-Owl }\end{array}$ & + & & & & & $\mathrm{E}$ & LC \\
\hline \multirow[t]{3}{*}{ Sturnidae } & $\begin{array}{l}\text { Acridotheres cristatellus } \\
\text { (Linnaeus, 1758) }\end{array}$ & Crested Myna & & + & + & + & + & 1 & $\mathrm{LC}$ \\
\hline & $\begin{array}{l}\text { Rhabdornis mystacalis } \\
\text { mystacalis } \\
\text { (Temminck, 1825) }\end{array}$ & $\begin{array}{l}\text { Stripe-headed Rhabdornis, } \\
\text { Stripe-headed Creeper, } \\
\text { Stripe-sided Rhabdornis }\end{array}$ & + & & & & & $\mathrm{E}$ & $\mathrm{LC}$ \\
\hline & $\begin{array}{l}\text { Sarcops calvus } \\
\text { (Linnaeus, 1766) }\end{array}$ & Coleto & + & & & & & $\mathrm{E}$ & $\mathrm{LC}$ \\
\hline Trogonidae & $\begin{array}{l}\text { Harpactes ardens } \\
\text { (Temminck, 1826) }\end{array}$ & Philippine Trogon & + & & & & & $\mathrm{E}$ & $\mathrm{LC}$ \\
\hline Turdidae & $\begin{array}{l}\text { Geokichla cinerea } \\
\text { (Bourns \& Worcester, } \\
\text { 1894) }\end{array}$ & Ashy Thrush & + & & & & & $E$ & VU \\
\hline
\end{tabular}


Philippine Journal of Systematic Biology | Lagat,R.D. \& Causaren,R.M.: Terrestrial vertebrate diversity assessment in upland Cavite

\begin{tabular}{|c|c|c|c|c|c|c|c|c|c|}
\hline Family & Taxa & English/Common name & $\mathbf{P}$ & I & G & A & $\mathbf{T}$ & ES & $\mathrm{CS}$ \\
\hline Turnicidae & $\begin{array}{l}\text { Turnix ocellatus (Scopoli, } \\
1786 \text { ) }\end{array}$ & Spotted Buttonquail & + & & & & & $E$ & LC \\
\hline Zosteropidae & $\begin{array}{l}\text { Zosterornis striatus } \\
\text { (Ogilvie-Grant, 1894) }\end{array}$ & Luzon Striped Babbler & + & & & & & $E$ & NT \\
\hline
\end{tabular}

\section{ANURANS}

\begin{tabular}{|c|c|c|c|c|c|c|c|c|c|}
\hline Bufonidae & $\begin{array}{l}\text { Rhinella marina } \\
\text { (Linnaeus, 1758) }\end{array}$ & $\begin{array}{l}\text { Giant South American } \\
\text { Toad }\end{array}$ & & + & & + & + & 1 & LC \\
\hline \multirow[t]{2}{*}{ Microhylidae } & $\begin{array}{l}\text { Kaloula picta } \\
\text { (Duméril and Bibron, } \\
1841 \text { ) }\end{array}$ & Slender-digit Chorus Frog & & & + & + & + & $E$ & LC \\
\hline & $\begin{array}{l}\text { Kaloula pulchra } \\
\text { Gray, } 1831\end{array}$ & $\begin{array}{l}\text { Malaysian Narrowmouth } \\
\text { Toad }\end{array}$ & & & & + & & 1 & LC \\
\hline \multirow[t]{7}{*}{ Ceratobatrachidae } & $\begin{array}{l}\text { Platymantis corrugatus } \\
\text { (Duméril, 1853) }\end{array}$ & Rough-backed Forest Frog & + & & & & & $E$ & LC \\
\hline & $\begin{array}{l}\text { Platymantis dorsalis } \\
\text { (Duméril, 1853) }\end{array}$ & $\begin{array}{l}\text { Dumeril's Wrinkled Ground } \\
\text { Frog }\end{array}$ & + & & & + & & $E$ & LC \\
\hline & Platymantis Iuzonensis & & & & & & & & \\
\hline & $\begin{array}{l}\text { Brown, Alcala and } \\
\text { Diesmos, } 1997\end{array}$ & Luzon Forest Frog & + & & & & & $E$ & NT \\
\hline & Platymantis mimulus & & & & & & & & \\
\hline & $\begin{array}{l}\text { Brown, Alcala and } \\
\text { Diesmos, } 1997\end{array}$ & Diminutive Forest Frog & + & + & + & + & + & $E$ & NT \\
\hline & Platymantis sp. & & + & & & & & $E$ & DD \\
\hline \multirow[t]{5}{*}{ Ranidae } & $\begin{array}{l}\text { Hylarana erythraea } \\
\text { (Schlegel, 1837) }\end{array}$ & Common Green Frog & & + & + & + & + & 1 & LC \\
\hline & $\begin{array}{l}\text { Limnonectes } \\
\text { macrocephalus } \\
\text { (Inger, 1954) }\end{array}$ & $\begin{array}{l}\text { Giant Philippine Frog, } \\
\text { Luzon Fanged Frog }\end{array}$ & + & + & + & + & + & $E$ & NT \\
\hline & $\begin{array}{l}\text { Limnonectes } \\
\text { woodworthi Taylor, } 1923\end{array}$ & Luzon Swamp Frog & + & + & & + & + & $E$ & LC \\
\hline & $\begin{array}{l}\text { Occidozyga laevis } \\
\text { (Günther, 1858) }\end{array}$ & Yellow-bellied Puddle Frog & + & + & & + & + & $\mathrm{N}$ & LC \\
\hline & $\begin{array}{l}\text { Pulchrana similis } \\
\text { (Günther, 1873) }\end{array}$ & Yellow-striped Stream frog & + & & & & & $E$ & NT \\
\hline \multirow[t]{2}{*}{ Rhacophoridae } & $\begin{array}{l}\text { Polypedates leucomystax } \\
\text { (Gravenhorst, 1829) }\end{array}$ & White-lipped Tree Frog & + & + & + & + & + & $\mathrm{N}$ & LC \\
\hline & $\begin{array}{l}\text { Rhacophorus pardalis } \\
\text { Günther, } 1859\end{array}$ & Panther Flying Frog & + & + & & & & $\mathrm{N}$ & LC \\
\hline \multicolumn{10}{|l|}{ LIZARDS } \\
\hline \multirow[t]{2}{*}{ Agamidae } & $\begin{array}{l}\text { Bronchocela cristatella } \\
\text { (Kuhl, 1820) }\end{array}$ & Green-crested lizard & + & & & & & $\mathrm{N}$ & NE \\
\hline & $\begin{array}{l}\text { Draco spilopterus } \\
\text { (Wiegmann, 1834) }\end{array}$ & Flying Dragon & + & + & & & & $E$ & NE \\
\hline
\end{tabular}




\begin{tabular}{|c|c|c|c|c|c|c|c|c|c|}
\hline Family & Taxa & English/Common name & $\mathbf{P}$ & 1 & $\mathbf{G}$ & A & $\mathrm{T}$ & ES & $\mathrm{CS}$ \\
\hline \multirow[t]{2}{*}{ Agamidae } & $\begin{array}{l}\text { Gonocephalus sophiae } \\
\text { (Gray, 1845) }\end{array}$ & Angled-head Lizard & + & & & & & $E$ & DD \\
\hline & $\begin{array}{l}\text { Hydrosaurus pustulatus } \\
\text { (Eschsholtz, 1829) }\end{array}$ & Sailfin Lizard & & & + & + & & $\mathrm{E}$ & VU \\
\hline \multirow[t]{6}{*}{ Gekkonidae } & $\begin{array}{l}\text { Cyrtodactylus } \\
\text { philippinicus } \\
\text { (Steindachner, 1867) }\end{array}$ & Philippine Bent-toed Gecko & + & + & + & + & + & $E$ & LC \\
\hline & $\begin{array}{l}\text { Gekko mindorensis } \\
\text { Taylor, } 1919\end{array}$ & $\begin{array}{l}\text { Mindoro Narrow-disked } \\
\text { Gecko }\end{array}$ & + & & & & + & $E$ & NE \\
\hline & $\begin{array}{l}\text { Gehyra mutilata } \\
\text { (Wiegmann, 1834) }\end{array}$ & $\begin{array}{l}\text { Stump-toed Gecko, } \\
\text { Common Four-clawed } \\
\text { Gecko, Stump-tailed Gecko }\end{array}$ & + & & & & & $\mathrm{N}$ & NE \\
\hline & $\begin{array}{l}\text { Gekko gecko (Linnaeus, } \\
1758 \text { ) }\end{array}$ & $\begin{array}{l}\text { Tokay Gecko, Tuctoo, } \\
\text { Tokeh-tokeh }\end{array}$ & + & + & + & + & + & $\mathrm{N}$ & NE \\
\hline & $\begin{array}{l}\text { Hemidactylus frenatus } \\
\text { Duméril \& Bibron, } 1836\end{array}$ & $\begin{array}{l}\text { Common House Gecko, } \\
\text { South Asian House Gecko, } \\
\text { Bridled House Gecko }\end{array}$ & + & & & & & I & NE \\
\hline & $\begin{array}{l}\text { Pseudogekko brevipes } \\
\text { (Boettger, 1897) }\end{array}$ & $\begin{array}{l}\text { Orange-Spotted } \\
\text { Smooth-Scaled Gecko }\end{array}$ & + & & & & + & $\mathrm{E}$ & VU \\
\hline \multirow[t]{11}{*}{ Scincidae } & $\begin{array}{l}\text { Brachymeles bonitae } \\
\text { Duméril \& Bibron, } 1839\end{array}$ & $\begin{array}{l}\text { Stub-limbed Burrowing } \\
\text { Skink }\end{array}$ & + & & & & & $\mathrm{E}$ & LC \\
\hline & $\begin{array}{l}\text { Brachymeles boulengeri } \\
\text { Taylor, } 1922\end{array}$ & $\begin{array}{l}\text { Boulenger's Short-legged } \\
\text { Skink }\end{array}$ & + & + & & & & $E$ & LC \\
\hline & $\begin{array}{l}\text { Brachymeles kadwa Siler } \\
\text { \& Brown, } 2010\end{array}$ & Jessi's Slender Skink & + & & & & + & $\mathrm{E}$ & NE \\
\hline & $\begin{array}{l}\text { Emoia atrocostata } \\
\text { (Lesson, 1830) }\end{array}$ & $\begin{array}{l}\text { Littoral Whiptail-Skink, } \\
\text { Mangrove Skink, Littoral } \\
\text { Skink }\end{array}$ & & & + & & & $\mathrm{N}$ & NE \\
\hline & $\begin{array}{l}\text { Eutropis multifasciata } \\
\text { (Kuhl, 1820) }\end{array}$ & $\begin{array}{l}\text { East Indian Brown Mabuya, } \\
\text { Many-lined Sun Skink, } \\
\text { Common Sun Skink, Javan } \\
\text { Sun Skink }\end{array}$ & + & & + & + & & $\mathrm{N}$ & NE \\
\hline & $\begin{array}{l}\text { Lamprolepis smaragdina } \\
\text { (Lesson, 1829) }\end{array}$ & $\begin{array}{l}\text { Emerald Skink, Green Tree } \\
\text { Skink }\end{array}$ & + & & & & + & $\mathrm{N}$ & NE \\
\hline & $\begin{array}{l}\text { Lepidodactylus lugubris } \\
\text { (Duméril \& Bibron, 1836) }\end{array}$ & $\begin{array}{l}\text { Mourning Gecko, Common } \\
\text { Smooth-Scaled Gecko }\end{array}$ & + & & & + & & $\mathrm{N}$ & $\mathrm{NE}$ \\
\hline & $\begin{array}{l}\text { Lipinia pulchella (Gray, } \\
\text { 1845) }\end{array}$ & Beautiful Lipinia & + & & & & & $\mathrm{E}$ & LC \\
\hline & $\begin{array}{l}\text { Eutropis cumingi (Brown } \\
\text { \& Alcala, 1980) }\end{array}$ & Cuming's Mabuya & + & + & & & & $\mathrm{E}$ & LC \\
\hline & $\begin{array}{l}\text { Eutropis multicarinata } \\
\text { borealis (Brown \& Alcala, } \\
1980 \text { ) }\end{array}$ & Many-keeled Mabuya & + & & & & & $\mathrm{N}$ & NE \\
\hline & $\begin{array}{l}\text { Eutropis multifasciata } \\
\text { (Kuhl, 1820) }\end{array}$ & $\begin{array}{l}\text { East Indian Brown Mabuya, } \\
\text { Many-lined Sun Skink, } \\
\text { Common Sun Skink, Javan } \\
\text { Sun Skink }\end{array}$ & + & & & & & $\mathrm{N}$ & $\mathrm{NE}$ \\
\hline
\end{tabular}


Philippine Journal of Systematic Biology | Lagat,R.D. \& Causaren,R.M.: Terrestrial vertebrate diversity assessment in upland Cavite

\begin{tabular}{|c|c|c|c|c|c|c|c|c|c|}
\hline Family & Taxa & English/Common name & $\mathbf{P}$ & 1 & G & A & $\mathbf{T}$ & ES & CS \\
\hline \multirow[t]{4}{*}{ Scincidae } & $\begin{array}{l}\text { Pinoyscincus jagori } \\
\text { (Peters, 1864) }\end{array}$ & Jagor's Sphenomorphus & + & + & & + & + & $E$ & LC \\
\hline & $\begin{array}{l}\text { Sphenomorphus cumingi } \\
\text { (Gray, 1845) }\end{array}$ & Cuming's Sphenomorphus & + & & & & & $E$ & LC \\
\hline & $\begin{array}{l}\text { Parvoscincus decipiens } \\
\text { (Boulenger, 1895) }\end{array}$ & $\begin{array}{l}\text { Black-sided } \\
\text { Sphenomorphus }\end{array}$ & + & & & & & $E$ & LC \\
\hline & $\begin{array}{l}\text { Parvoscincus steerei } \\
\text { (Stejneger, 1908) }\end{array}$ & Steere's Sphenomorphus & + & & & & & $E$ & LC \\
\hline \multirow[t]{2}{*}{ Varanidae } & $\begin{array}{l}\text { Varanus salvator } \\
\text { (Laurenti, 1768) }\end{array}$ & Common Water Monitor & + & + & & & & $\mathrm{N}$ & LC \\
\hline & $\begin{array}{l}\text { Varanus olivaceus } \\
\text { Hallowell, } 1859\end{array}$ & $\begin{array}{l}\text { Gray's Monitor, } \\
\text { Gray's Monitor Lizard }\end{array}$ & + & & & & & $E$ & VU \\
\hline
\end{tabular}

\section{MAMMALS}

\begin{tabular}{|c|c|c|c|c|c|c|c|c|c|}
\hline \multirow[t]{5}{*}{ Pteropodidae } & $\begin{array}{l}\text { Cynopterus brachyotis } \\
\text { (Müller, 1838) }\end{array}$ & $\begin{array}{l}\text { Common Short-nosed } \\
\text { Fruit Bat }\end{array}$ & + & & + & + & & W & LC \\
\hline & $\begin{array}{l}\text { Macroglossus minimus } \\
\text { (É. Geoffroy, 1810) }\end{array}$ & $\begin{array}{l}\text { Dagger-toothed } \\
\text { Long-nosed Fruit Bat }\end{array}$ & + & & + & & & $\mathrm{N}$ & LC \\
\hline & Otopteropus & & & & & & & & \\
\hline & $\begin{array}{l}\text { cartilagonodus Kock, } \\
1969\end{array}$ & Luzon Pygmy Fruit Bat) & + & & + & & & LE & LC \\
\hline & $\begin{array}{l}\text { Ptenochirus jagori } \\
\text { (Peters, 1861) }\end{array}$ & Musky fruit bat & + & + & & + & & $E$ & LC \\
\hline \multirow[t]{2}{*}{ Rhinolophidae } & $\begin{array}{l}\text { Rhinolophus subrufus } \\
\text { Andersen, } 1905\end{array}$ & $\begin{array}{l}\text { Small Rufous Horseshoe } \\
\text { Bat }\end{array}$ & + & + & & & & $E$ & DD \\
\hline & $\begin{array}{l}\text { Rhinolophus virgo } \\
\text { Andersen, } 1905\end{array}$ & $\begin{array}{l}\text { Yellow-faced Horseshoe } \\
\text { Bat }\end{array}$ & + & & + & & & $E$ & LC \\
\hline Cercopithecidae & $\begin{array}{l}\text { Macaca fascicularis } \\
\text { (Raffles, 1821) }\end{array}$ & Phil. long-tailed Macaque & + & & + & & + & $\mathrm{N}$ & NT \\
\hline \multirow[t]{2}{*}{ Muridae } & $\begin{array}{l}\text { Phloeomys cumingi } \\
\text { (Waterhouse, 1839) }\end{array}$ & $\begin{array}{l}\text { Southern Luzon } \\
\text { Giant Cloud Rat }\end{array}$ & & + & & & + & LE & VU \\
\hline & $\begin{array}{l}\text { Rattus everetti (Günther, } \\
\text { 1879) }\end{array}$ & $\begin{array}{l}\text { Common Philippine Forest } \\
\text { Rat }\end{array}$ & + & & + & + & & $E$ & LC \\
\hline Soricidae & $\begin{array}{l}\text { Crocidura grayi Dobson, } \\
1890\end{array}$ & Luzon Shrew & + & + & + & + & + & $E$ & LC \\
\hline Suidae & $\begin{array}{l}\text { Sus philippensis Nehring, } \\
1886\end{array}$ & Philippine Warty Pig & + & & & & & $E$ & VU \\
\hline Viverridae & $\begin{array}{l}\text { Paradoxurus } \\
\text { hermaphroditus } \\
\text { philippinensis } \\
\text { (Pallas 1777) }\end{array}$ & Common Palm Civet & + & + & + & + & + & $E$ & LC \\
\hline $\begin{array}{l}\text { Total number } \\
\text { of species }\end{array}$ & Total number of species & Total number of species & 157 & 42 & 40 & 48 & 46 & & 175 \\
\hline
\end{tabular}

The IUCN Red List of Threatened Species. Version 2017-2. <www.iucnredlist.org>. Downloaded on 17 October 2017. 


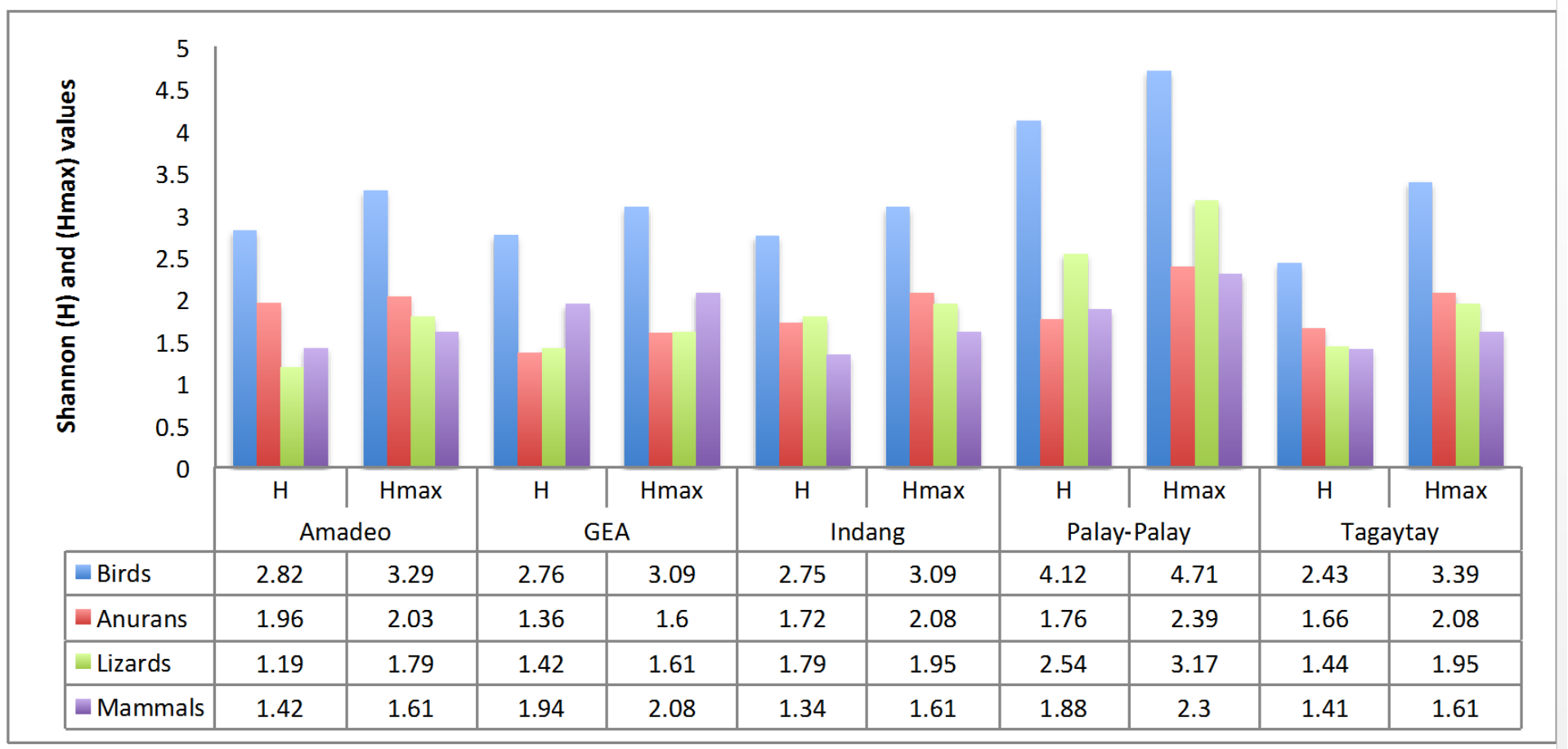

Figure 3. Species diversity index values of the major terrestrial fauna of upland Cavite.

observation can be attributed to the available habitats and resources that can support more species and bigger populations. On the contrary, small habitat fragments have limited resources and higher competition (Lawrence et al. 2018). In addition, the degree of anthropogenic-related disturbances in all the study sites is more pronounced in smaller habitat fragments that continuously reduce habitat availability as well as suitability for wildlife (Mullu 2016). Mt. Palay-Palay is protected under the NIPAS law, however, the other forest fragments are still at risk of being totally destroyed.

\section{Local Conservation Status}

Based on the latest IUCN updates on conservation status, 11 bird species (Mearnsia picina, Ducula poliocephala, Gallicolumba Iuzonica Iuzonica, Ptilinopus merrilli, Irena cyanogastra, Lanius validirostris, Hypothymis helenae, Cyornis herioti, Mulleripicus funebris, Otus longicornis, and Zosterornis striatus) recorded in the area are with NearThreatened (NT) status. Eight "Vulnerable (VU)" bird species include the North Philippine dwarf-kingfisher (Ceyx melanurus), Philippine duck (Anas luzonica), Northern rufous hornbill (Buceros hydrocorax), black-bibbed cuckoo-shrike (Edolisoma mindanense lecroyae), spotted imperial-pigeon (Ducula carola), great slaty woodpecker (Mulleripicus pulverulentus), and the Philippine eagle-owl (Bubo philippensis). Except for $C$. herioti and Anas luzonica which were also observed in GEA, all the remaining 17 species with NT and VU status were observed only in Mt. Palay-Palay. The absence of these species in other smaller and more disturbed fragments shows that most likely the remaining populations of these birds are confined within Mt. Palay-Palay where suitable habitats are still present. Considering that the remaining good forest cover is only about 640 ha, these species can be considered as locally threatened in the remaining forests of Cavite. Two mammals are listed as VU: Southern Luzon giant cloud rat (Phloeomys cumingi) and Philippine warty pig (Sus philippensis). The common justifications for these are restricted/limited population distribution and rapid population decline due to habitat loss/ degradation compounded by hunting pressures (IUCN 2017).

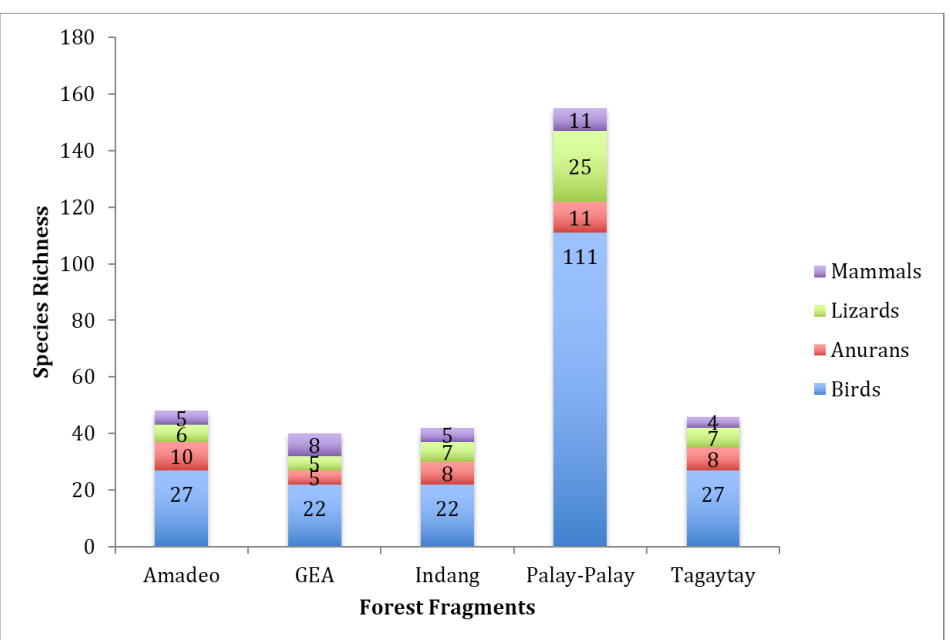

Figure 4. Species richness distribution of the different terrestrial vertebrate group per forest fragment 

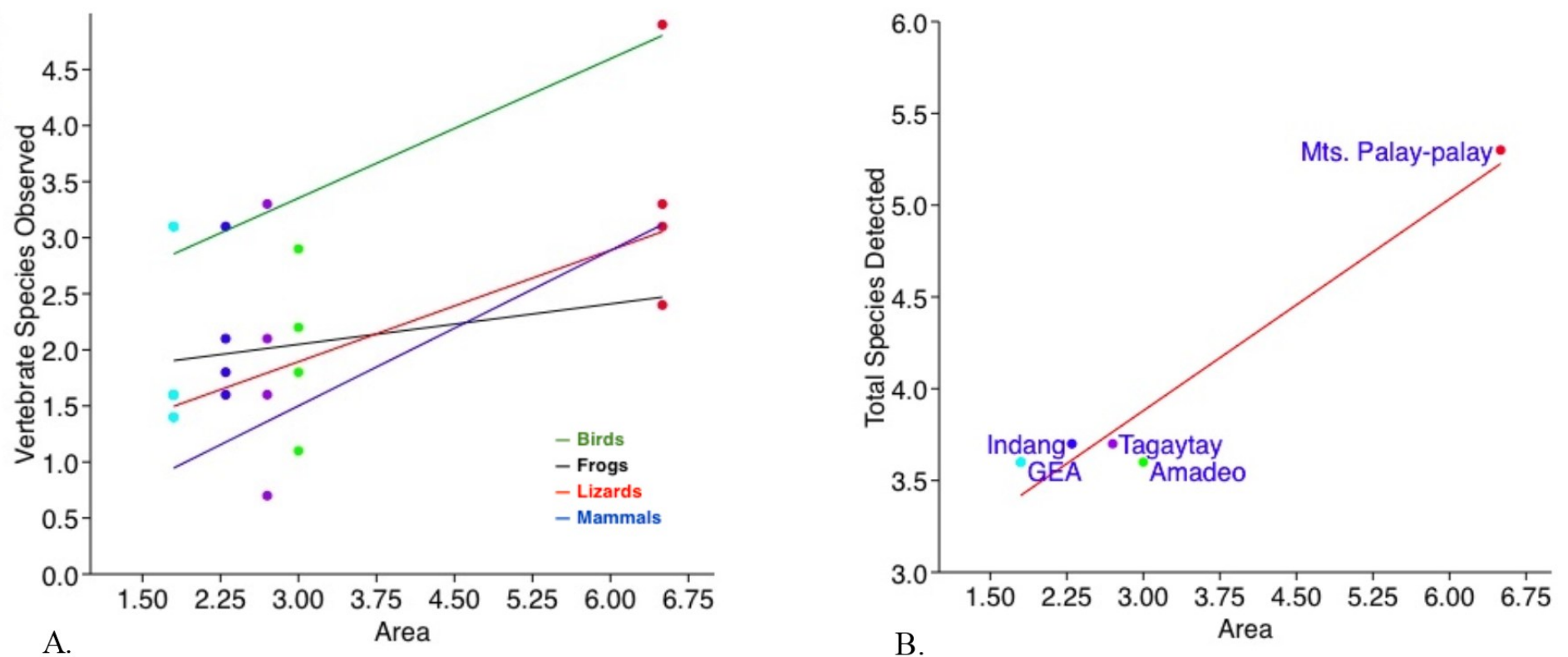

Figure 5. Linear fit plots generated from PAST3.22 (Hammer 2018). A) Positive linear relationships were observed with the speciesarea distribution for all the terrestrial vertebrate taxa included. B) Total species-area distribution having positive linear relation in all the study areas.

Based from confidence interval distribution at 0.05a (Figure 6), other "VU" species include three lizards: Sailfin Lizard (Hydrosaurus pustulatus, observed only in Indang), Orangespotted Smooth-scaled Gecko (Pseudogekko brevipes) and Gray's Monitor Lizard (Varanus olivaceus). V. olivaceus is the first record of a frugivorous lizard reported from western Luzon (Welton et al. 2012; Figure 7) and $P$. brevipes is the only pseudo-gecko in Cavite, both species were observed only in Mt. Palay-Palay. Sailfin Lizard (Hydrosaurus pustulatus, VU, IUCN 2017), which was observed only in Indang and Mt. Palay-Palay requires special habitats (sandy river banks and lush riparian vegetation) for egg-laying and foraging. Its habitats are also shrinking specially in Indang due to conversion of forest to agricultural and residential areas. These three species based on the actual detection in the study areas reflect the most limited distribution which ranges from 0.02 to 0.37 confidence interval (Figure 6). However, Gonocephalus sp. which is not evaluated (NE) in IUCN (2017) also was documented with one of the lowest distributions in the area (Figure 6). Restricted local distribution and continuous habitat loss will qualify all these lizards as locally threatened in Cavite. Forest restricted species such as $B$. bonitae, B. boulengeri, B. kadwa, $L$. lugubris, L. pulchella, E. cumingi, E. multicarinata, $S$.

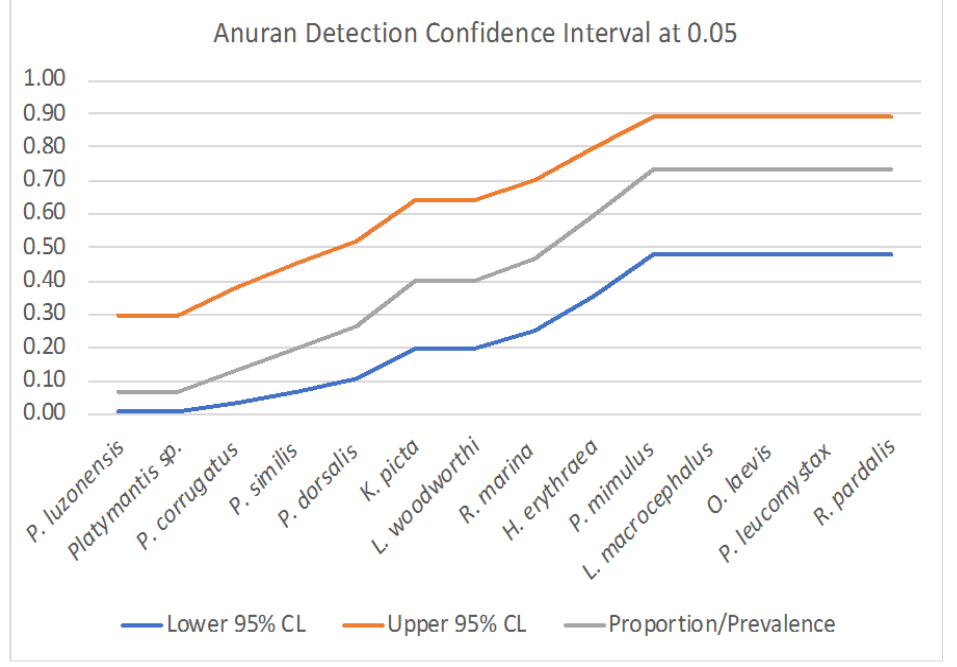

A

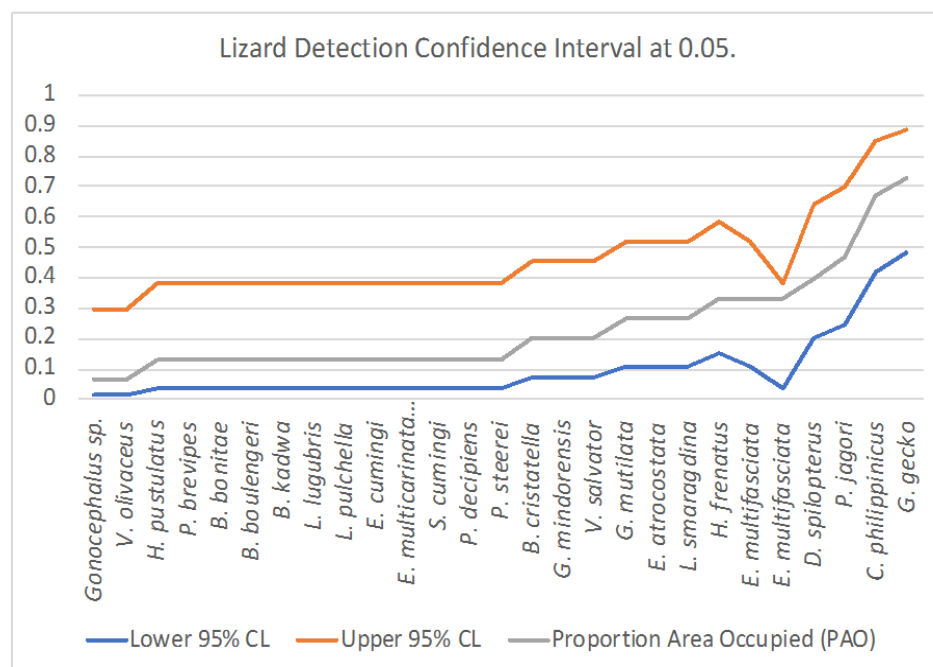

B

Figure 6: Detection confidence distributions of anurans (A) and lizards (B) in Cavite. 


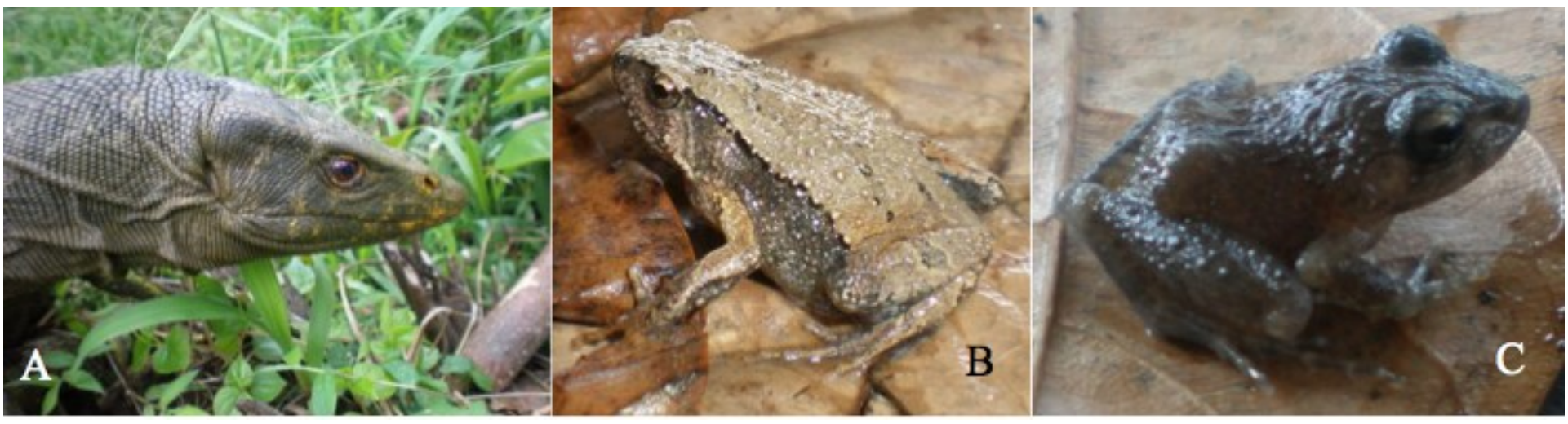

Figure 7. Some notable terrestrial vertebrate species. A. Varanus olivaceus, B. Kaloula sp., C. Platymantis sp.

cumingi, $P$. decipiens and $P$. steerei with computed confidence interval distribution of no more than 0.37 (Figure 6) are locally VU also due to restricted distribution and continuous habitat loss.

Two anurans species, Platymantis sp. and Kaloula sp. (Figure 7 ), are currently undescribed and possibly endemic to the province. Platymantis $\mathrm{sp}$. together with $P$. luzonensis registered the lowest confidence interval distribution of 0.07 to 0.30 range (Figure 6 ). For having a restricted distribution and being forest obligates, these two species can be considered as locally threatened anuran species in Cavite.

\section{Major Anthropogenic Threats}

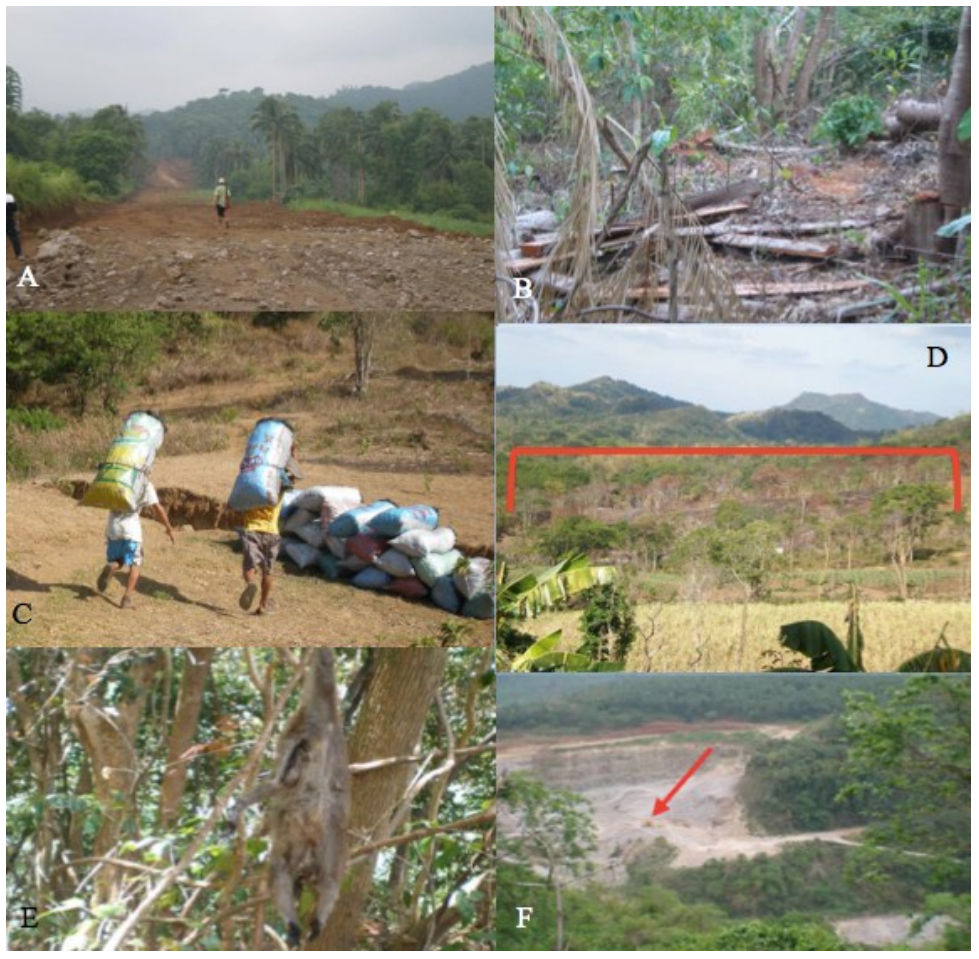

Figure 8. Some anthropogenic threats documented. Road construction (A), illegal logging (B), charcoal making $(\mathrm{C})$, kaingin (D), poaching $(E)$, and quarrying (red arrow points to a bulldozer, F). (Photos by R.D. Lagat and R.M. Causaren)
Several threats (conversion of forest habitats to agricultural or residential areas, illegal logging, poaching, firewood harvesting, charcoal making, road construction and, quarrying) were documented during field sampling activities (Figure 8). All of these activities were observed in Mt. PalayPalay. Except for illegal logging and quarrying, the other threats were also observed in GEA, Amadeo, Indang, and Tagaytay. Conversion of forest habitats to agricultural farms or residential areas was the most common threat and can be considered as the most significant since this resulted in habitat loss.

All of these threats contribute to habitat loss. From activities that have a direct impact on wildlife populations (illegal logging and poaching) to complete habitat destruction (quarrying, road construction, and charcoal making) negatively affect local biodiversity. Agricultural related activities (farming and animal raising) also contribute to habitat loss due to land conversion and the associated pollution (water pollution resulting from synthetic fertilizers and pesticides use). The frequency, distribution, and proportion of area occupied (PAO) of the different species considering endemicity, forest association and disturbance tolerance and the existing threats reflect the state of wildlife habitats respectively (Landres et al. 1988; Siddig et al. 2016). Forest restricted species mainly endemic and native in distribution were observed in areas where relatively good forest habitat still remain in Mt. Palay-Palay. As all threats earlier enumerated are observed in this area, the presence of introduced, disturbance tolerant and invasive terrestrial vertebrate species are evident. More developed areas (Tagaytay, Indang, Amadeo and GEA) reflect significant environmental disturbance as shown by a greater proportion of non-native and disturbance tolerant terrestrial vertebrate species. On the other hand, few fragments of natural forest habitat still remain in these areas as a number of forestrestricted terrestrial vertebrate species are still present (Table 1). Terrestrial vertebrate species already evaluated with vulnerable (VU) and near-threatened (NT) status can be locally considered as critically endangered considering the 
continuous habitat degradation brought about by further industrial development of Cavite.

All observed threats are anthropogenic in nature. In line with the Philippine Wildlife Act of 2001 (R. A. 9147), the Expanded National Integrated Protected Area System Act of 2018 (R. A. 11038) and Ecological Solid Waste Management Act of 2000, related environmental problems can be addressed by strict adherence to these laws. Local community-based conservation programs should be initiated so as to build capacity and directly engage the locals in protecting and managing wildlife habitats. Alternative livelihood programs such as environmental tourism in Tagaytay, Malibiclibic falls in GEA, river resorts in Amadeo and Eco-trekking in Mt. Palay -Palay can generate economic activities that will allow the locals to earn without directly harming the environment.

To appropriately address the identified environmental problems, Life Cycle Analysis (LCA) studies should be undertaken to fully understand the nature and impact of these problems. Carefully calibrated LCA's can significantly contribute to sound policies and actions that will properly address specific issues and concerns (Jensen et al. 1997).

\section{CONCLUSIONS AND RECOMMENDATIONS}

Relatively, higher terrestrial vertebrate diversity was observed in areas where the availability of less disturbed forest habitats was documented such as in Mt. Palay-Palay. Among the terrestrial vertebrate groups, the birds had the highest observed diversity and distribution. Species-area relationships showed positive correlations. Forest restricted species which showed limited distribution exacerbated with continuous habitat loss were assessed as locally threatened.

This study only provides an initial assessment of the terrestrial vertebrates in some remaining forest fragments in Cavite. A more extensive field sampling, especially for birds and mammals, are warranted. The data generated can be used in the formulation of an effective biodiversity management and conservation plan and hopefully strengthen legislation in relation to habitat and species protection.

\section{ACKNOWLEDGEMENT}

We are grateful to Mr. Edilberto Perido, Ricky Morales, Dr. Melanie Guiang, Ms. Myra Lagat, and Mr. Alvin F. Laurio for their valuable assistance during the study. Protected Area Management Board and staff of Mt. Palay-Palay and the Department of Environment and Natural Resources Region IV provided important technical support. We thank the University Research Office of De La Salle University-Dasmarinas for providing funding for this research.

\section{LITERATURE CITED}

Alcala, A.C., 1986. Guide to Philippine Flora and Fauna, Vol. $X$, Amphibians and Reptiles. Natural Resource Management Center and Ministry of Natural Resources and University of the Philippines, Quezon City, Philippines.

Alcala, A.C., \& W.C. Brown, 1998. Philippine Amphibians: an Illustrated Field Guide. Bookmark Press, Makati City, Philippines.

Alcala, E.L., A.C. Alcala, \& C. N. Dolino, 2004. Amphibians and reptiles in tropical rainforest fragments on Negros Island, the Philippines. Environmental Conservation, 31 (3):254-261.

Bibby, C. J., N. D. Burgess, D.A. Hill, \& S. H. Mustoe, 2000. Bird Census Techniques, 2nd ed. Academic Press, London.

Brown, W. C., A. C. Alcala, \& A. C. Diesmos, 1997a. A new species of the genus Platymantis (Amphibia: Ranidae) from Luzon Island, Philippines. Proceedings of the Biological Society of Washington,110:18-23.

Brown, W. C., A. C. Alcala, A. C. Diesmos, \& E. Alcala, 1997b. Species of the güntheri group of Platymantis with descriptions of four new species. Proceedings of the California Academy of Sciences, 50:1-20.

Brown, W.C., R. M. Brown, \& A.C. Alcala, 1997c. Species of the hazelae group of Platymantis (Amphibia: Ranidae) from the Philippines, with descriptions of two new species. Proceedings of the California Academy of Sciences, 49:405-421.

Brown, R. M., A.C. Diesmos, \& A.C. Alcala, 2001. The state of Philippine herpetology and the challenges for the next decade. Silliman Journal, 42:18-87.

Buckland, S.T., D.R. Anderson, K.P. Burnham, J.L. Laake, \& D.L. Borchers, 2001. Introduction to Distance Sampling: Estimating Abundance of Biological Populations. Oxford University Press, New York.

Buenaventura JA, Edles MB, Tomo VB. 2003. Documentation of fauna in Mts. Palaypalay-Mataas na Gulod National Park, Luzon Island, Philippines [undergraduate thesis]. [Cavite (PH)]: De La Salle University-Dasmariñas.

Bury, R. B., \& M. G. Raphael, 1983. Inventory methods for amphibians and reptiles. In J. F. Bell and T. A. Atterbury (eds.), Renewable Resource Inventories for Monitoring Changes and Trends: Proc. Internat. Conf., pp. 416-419. Soc. Am. For. 83-14.

Campbell, H. W. \& S. P. Christman, 1982. Field techniques for herpetofaunal community analysis. In N. J. Scott, Jr. (ed.), Herpetological Communities, pp. 193-200. U.S. Fish And Wildlife Service 13

Causaren, R.M., 2009. Preliminary report on the anurans of Mts. Palay-Palay Mataas-na-Gulod Protected Landscape, Luzon Island, Philippines. Philippine Journal 
Philippine Journal of Systematic Biology | Lagat,R.D. \& Causaren,R.M.: Terrestrial vertebrate diversity assessment in upland Cavite

of Systematic Biology, 3:40-56.

Causaren, R.M., 2012. Determinants of species diversity, abundance and habitat associations of the anuran fauna from forest fragments in Cavite, Luzon Island, Philippines. [Dissertation]. [Manila (PH)]: De La Salle University.

Causaren, R.M., 2016. Species diversity, abundance and habitat distribution of anurans in Mts. Palay-Palay Mataas -na-Gulod Protected Landscape, Luzon Island, Philippines. Philippine Journal of Systematic Biology, 10:63-76.

Causaren, R.M., A.C. Diesmos, \& N. A. Mallari, 2016. Anuran diversity and ecology from forest fragments in Cavite Province, Luzon Island, Philippines. Philippine Journal of Systematic Biology, 10:52-62.

Causaren, R.M., N.A. Mallari, \& A.C. Diesmos, 2017. Habitat associations of the anuran fauna from the forest fragments in Cavite, Luzon Island, Philippines. NMP Journal of Natural History, 2(1):11-24.

Celis, M.O., R.S. Crisostomo, \& O. A. Untivero, 1997. A survey of amphibian fauna in Mts. Palay-Palay/Mataas na Gulod, Ternate, Cavite. Cavite, Philippines: De La Salle University-Dasmariñas: Undergraduate Thesis.

Chaudhary, A. \& A.O. Mooers, 2018. Terrestrial vertebrate biodiversity loss under future Global land use change scenarios. Sustainability, 10: 1-20. (doi:10.3390/ su10082764)

Collar, N.J., M.J. Crosby, \& A. J. Stattersfield, 1994. Birds to Watch 2: The World List of Threatened Birds. Conservation Series No. 4: Birdlife International, Cambridge, United Kingdom.

Collar, N.J., N.A.D. Mallari, \& B.R.Tabaranza Jr., 1999. Threatened Birds of the Philippines. Haribon/BirdLife and Bookmark Inc. Makati City.

Colwell, R. K. 2016. EstimateS: statistical estimation of species richness and shared species from samples. Version 9.1.0. Available from: http:// viceroy.eeb.uconn.edu/estimates/

Crump, M., \& N.J. Scott, 1994. Visual encounter surveys. Pages 84-92 In: Heyer, W.R., \& M.A. Donnelly, R.W. McDiarmid, L.A.C. Hayek, \& M. S. Foster (eds.), Measuring and Monitoring Biological Diversity: Standard Methods for Amphibians. Smithsonian Institution Press, Washington, D.C., USA.

Cuevas, C.Z. 2016. Ecological and anthropogenic factors affecting bird and bat diversity in Mts. Palay-Palay/Mataas -na-Gulod Protected Landscape, Luzon, Philippines. Cavite, Philippines: De La Salle University-Dasmariñas: Doctoral Dissertation.

Department of Environment and Natural Resources (DENR), 1992. Master Plan for Forest Development, Manila.

Dickinson, E.C., R.S. Kennedy, \& K.C. Parkes, 1991.The birds of the Philippines, an annotated checklist.12:1-507.

Diesmos, A.C., 1998. The amphibian faunas of Mt. Banahao, Mt. San Cristobal, and Mt. Maquiling, Luzon Island,
Philippines. Laguna, Philippines: University of the Philippines at Los Baños College: Master's Thesis.

Diesmos, A.C., 1999. Frogs of Mt. Maquiling and Mt. Banahao. Rapid Color Guide \#51 version 1.0 October 1999.

Diesmos, A.C., 2008. Ecology and diversity of herpetofaunal communities in a fragmented lowland rainforest in the Philippines. Singapore: National University of Singapore: Dissertation.

Diesmos, A.C., R.M. Brown, \& A.C. Alcala, 2002. New species of narrow-mouthed frog (Amphibia: Anura; Microhylidae; genus Kaloula) from the mountains of southern Luzon and Polillo islands, Philippines. Copeia 4,:1037-1051.

Diesmos, A.C.\& the Herpetofauna Working Group, 2000. Philippine amphibians and reptiles:an overview of diversity, biogeography and conservation. Paper presented at the National Biodiversity Conservation Priority Setting Workshop, White Rock Hotel, Subic, Philippines.

Environmental Science for Social Change. '2002 Forest Cover of the Philippines' [map]. 1:250,000. 2002 Forest Cover of the Philippines Series Number 2514. Available from: http://essc.org.ph/component/ option,com_wrapper/ltemid,109/. Accessed March 2010.

Fisher, T. \& Hicks N., 2001. A Photographic Guide to Birds of the Philippines. Holland Australia: New Holland Australia. Food and Agriculture Organization of the United Nations (FAO), 2010. Global Forest Resources Assessment 2010 - Key Findings. Rome, Italy: Food and Agriculture Organization of the United Nations.

Frost, D.R., 2011. Amphibian Species of the World: an Online Reference. Version 5.5 (31 January, 2011). Electronic Database accessible at http:// research.amnh.org/vz/ herpetology/amphibia/American Museum of Natural History, New York, USA.

Guyamin, M.C., 2004. Terrestrial vertebrate assessment of Mts. Palay-Palay/Mataas na Gulod National Park, Luzon Island, Philippines [UFRO- approved research]. [Cavite $(\mathrm{PH})$ ]: De La Salle University-Dasmariñas.

Hammer O., D.A.T. Harper, \& P.D. Ryan, 2001. Paleontological Statistics (PAST) software v2.10 package for education and data analysis. Available from: http://www.folk.uio.no/ohammer/past. Downloaded on August 2011.

Heaney, L.R., D.S. Balete, M.L. Dolar, A.C. Alcala, A.T.L. Dans, P.C. Gonzales, N.R. Ingle M.V. Lepiten, W.L.R. Oliver, P.S. Ong, E.A. Rickart, B.R. Tabaranza, \& R.C.B. Utzurrum, 1998. A synopsis of the mammalian fauna of the Philippine Islands. Fieldiana Zoology, New Series, 88:1-61.

Heaney, L.R. \& N.A.D. Mallari, 2001. A preliminary analysis of the current gaps in the protection of threatened Philippine terrestrial mammals. Sy/vatrop, 10 (1 \& 2):2839. 
Philippine Journal of Systematic Biology | Lagat,R.D. \& Causaren,R.M.: Terrestrial vertebrate diversity assessment in upland Cavite

Heaney, L.R., L.R. Walker, B.R. Tabaranza, N.R. Ingle, 2000. Mammalian diversity in the Philippines: an assessment of the adequacy of current data. Sy/vatrop, 10(1\&2):6-27.

Heaney, L.R., \& E.A. Rickart, 2016. The Mammals of Luzon Island: Biogeography and Natural History of a Philippine Fauna. Baltimore, Maryland: Johns Hopkins University Press.

Heaney, L.R., M.L. Dolar, D.S. Balete, J.A. Esselstyn, E.A. Rickart \& J.L. Sedlock, 2010. Synopsis of Philippine Mammals. (The Field Museum) Retrieved October 10, 2017, from Synopsis of Philippine Mammals: https:// www.fieldmuseum.org/synopsis-philippine-mammals

Heyer, W.R., M.A. Donnelly, R.W. McDiarmid, L.C. Hayek, M.S. Foster, 1994. Measuring and Monitoring Biological Diversity: Standard Methods for Amphibians. Smithsonian Institution Press. pp.41-104, 275-279.

IUCN Red List of Threatened Species. Version 2017-2. <www.iucnredlist.org>. Downloaded on 17 October 2017.

Jacinto, W.R. \& R.B. America, 2005. Diversity of fishes of Maragondon River, Mts. Palay-Palay Mataas na Gulod, Luzon Philippines [UFRO- approved research]. [Cavite $(\mathrm{PH})$ ]: De La Salle University-Dasmariñas.

Jackson, H. B. \& L. Fahrig, 2013. Habitat loss and fragmentation. Elsevier: Carleton University, Ottawa, ON, Canada.

Jenkins, C.N., S. L., Pimm, \& L.N. Joppa, 2013. Global patterns of terrestrial vertebrate diversity and conservation. Proceedings of the National Academy of Sciences, 110(28):1-9. (www.pnas.org/cgi/doi/10.1073/ pnas.1302251110)

Jensen, A. A., L. Hoffman, B. T. Moller, A. Schmidt, K. Christiansen, \& J. Elkington, 1997. Life Cycle Assessment (LCA) A guide to approaches, experiences and information sources. United Kingdom: Sustainability, United Kingdom.

Lagat, R.D., 1999. Distribution patterns and observations on the influence of environmental factors on selected lizard species of Mts. Palay Palay-Mataas na Gulod National Park, Luzon Island, Philippines. Cavite, Philippines: De La Salle University-Dasmariñas: Masteral Thesis.

Lagat, R.D., 2009. A Taxonomic account of lizards along established trails in Mts. Palay-Palay Mataas-na-Gulod Protected Landscape, Luzon Island, Philippines. Philippine Journal of Systematic Biology, 3:17-28.

Lagat, R.D., 2012. Determinants of species diversity and occupancy of lizard fauna in the remaining forest patches of Cavite, Luzon Island, Philippines. Manila, Philip- pines: De La Salle University: Unpublished PhD Dissertation.

Lalap, N.A.M. \& L.S. Ybanez, 2006. Population distribution patterns of bird species in Mts. Palay-palay/Mataas na Gulod National Park, Luzon Island, Philippines [undergraduate thesis]. [Cavite (PH)]: De La Salle University-Dasmariñas.

Landres, P. B., J. Verner, \& J. W. Thomas, 1988. Ecological uses of vertebrate indicator species: a critique. Conservation Biology, 2(4): 316-328.

Lardizabal, L.M.C. \& R.T. Maniago, 1996. A survey of rodents found in Mts. Palay-Palay/ Mataas na Gulod National Park, Ternate, Cavite [undergraduate thesis]. [Cavite $(\mathrm{PH})$ ]: De La Salle University-Dasmariñas.

Lawrence, A., O'Connor, K., Haroutounian, V., \& A. Swei. 2018. Patterns of diversity along a habitat size gradient in a biodiversity hotspot. Ecosphere, 9(4):e02183. 10.1002/ecs2.2183.

Liu, D. S., L.R. Iverson, \& S. Brown, 1993. Rates and patterns of deforestation in the Philippines: application of geographic information system analysis. Forest Ecology and Management, 57:1-16.

Lo, E.P., \& M. L. O. Quemuel, 1998. Survey of bats at Mts. Palay-Palay/Mataas na Gulod National Park, Ternate, Cavite [undergraduate thesis]. [Cavite (PH)]: De La Salle University-Dasmariñas.

Lope, D.J.C. \& G.O. Hernandez, 2008. Elevational assessment of wild rodents found in Pico de Loro of Mts. Palaypalay-Mataas na Gulod National Park, Luzon Island, Philippines [undergraduate thesis]. [Cavite $(\mathrm{PH})$ ]: De La Salle University-Dasmariñas.

Lorenzana, C.M.G. \& M. D. C. Rocamora, 1997. An avifaunal survey of Mts. Mts. Palay-Palay/Mataas na Gulod National Park, Ternate, Cavite [undergraduate thesis]. [Cavite (PH)]: De La Salle UniversityDasmariñas.

MacArthur, R., \& E. O. Wilson. 1967. The theory of island biogeography. New Jersey: Princeton University Press.

MacKenzie, D.I., J.D. Nichols, G.B. Lachman, S. Droege, J.A. Royle, \& C.A. Langtimm, 2002. Estimating site occupancy rates when detection probabilities are less than one. Ecology, 83(3): 2248-2255.

Mackenzie, D.I., J.D. Nichols, J.A. Royle, K. Pollock, L. Bailey, \& J.E. Hines, 2006. Occupancy Estimation and Modeling - Inferring Patterns and Dynamics of Species Occurrence. Elsevier Publishing. 343 pp.

Mallari, N.A.D. \& the Bird Working Group, 2000. Philippine Birds: Setting an Agenda for Conservation. Paper presented at the National Biodiversity Conservation Priority Setting Workshop, White Rock Hotel, Subic, Philippines.

Mallari, N.A.D., B.R. Tabaranza Jr., \& M.J. Crosby, 2001. Key Conservation Sites in the Philippines: A Haribon Foundation and BirdLife International Directory of Important Bird Areas. Bookmark, Inc., Makati City, Philippines. 485 pp.

Maranan, R.L., 1999. Distribution patterns of the anurans of Mts. Palay-palay/Mataas na Gulod National Park, Luzon Island, Philippines. Manila, Philippines: De La Salle University: Masteral Thesis.

Medecilo, M.P., \& M.N. Lagat, 2017. Floristic composition of the remaining forests in Upland Cavite, Philippines. 
Philippine Journal of Systematic Biology | Lagat,R.D. \& Causaren,R.M.: Terrestrial vertebrate diversity assessment in upland Cavite

Philippine Journal of Systematic Biology, 11(1):74-94.

Medecilo, M. \& H. Luyon, 2006. An analysis of the dipterocarp vegetation of Mts. Palay- palay/Mataas Na Gulod National Park, Luzon Island, Philippines. Sinag, 10(2): 121-147.

Mittermeier, R.A., G.P. Robles, \& C.G. Mittermeier, 1997. Megadiversity, Earth's biologically wealthiest nations. CEMEX, Monterrey, Mexico. 501 pp.

Mullu, D. 2016. A review on the effect of habitat fragmentation on ecosystem . Journal of Natural Sciences Research, 6 (15): $1-15$.

Myers, N, R.A. Mittermeier, C.G. Mittermeier, G.A.B. da Fonseca, \& J. Kent, 2000. Hotspots: Earth's biologically richest and most endangered terrestrial ecoregions. Nature, 403: 853-858.

O'Farrell, M. J., W.A. Clark, F.H. Emmerson, S.M. Juarez, F.R. Kay, T.M. O'Farrell, \& T.Y. Goodlet, 1994. Use of a mesh live trap for small mammals: are results from Sherman live traps deceptive? Journal of Mammalogy, 75: 692-699.

Ong, P.S., L.E. Afuang, R.G. Rosell-Ambal, 2002. Philippine Biodiversity Conservation Priorities: A Second Iteration of the National Biodiversity Strategy and Action Plan. Department of Environment and Natural ResourcesProtected Areas and Wildlife Bereau, Conservation International Philippines, Biodiversity Conservation Program-U.P. Center for Integrated Development Studies, and Foundation for the Philippine Environment, Quezon City, Philippines.

Paloma, M.C.S., \& E.M. Panganiban, 1998. Herpetofauna found in Mts. Palay- palay/Mataas na Gulod, Ternate, Cavite. Cavite, Philippines: De La Salle UniversityDasmariñas: Undergraduate Thesis.

Pan, S. 2013. Fundamental equations for species-area theory. Scientific Reports, 3:1334.

Pancho, J., 1983. Vascular Flora of Mt. Makiling and Vicinity (Luzon, Philippines). Kalikasan, The Philippine Journal of Biology Supplement No.1.University of the Philippines at Los Baños.

Philippine Biodiversity Symposium (PBS), 2014. Proceeding from the $23^{\text {rd }}$ Annual Philippine Biodiversity Symposium held in the University of San Carlos, Talamban Campus, Cebu City on April 1-4, 2014.

Plumptre, A.J. \& V. Reynolds, 1994 The impact of selective logging on the primate populations in the Budongo Forest Reserve, Uganda. Journal of Applied Ecology, 31: 631641.

Preston, F. W. 1960. Time and space and the variation of species. Ecology 41:611-27.

Preston, F. W. 1962. The canonical distribution of commonness and rarity. Ecology, 43:185-215, 410-432.

Rademaekers, K., L. Eichler, J. Berg, M. Obersteiner, \& P. Havlik, 2010. Study on the Evolution of Some Deforestation Drivers and their Potential Impacts on the Costs of an Avoiding Deforestation Scheme. European
Commission Directorate-General for Environment Final Report. ECORYS Nederland.

Raroque, L.L. \& B.V. Valerio, 1999. A study on the foraging pattern and selected Pteropodidae bats (Mammalia:Chiroptera) on Mts. Mts. Palay-Palay/ Mataas na Gulod National Park, Ternate, Cavite [undergraduate thesis]. [Cavite (PH)]: De La Salle University-Dasmariñas.

Schmitt C.J., J. A. Cook, K.R. Zamudio, \& S.V. Edwards, 2018. Museum specimens of terrestrial vertebrates are sensitive indicators of environmental change in the Anthropocene. Philosophical Transactions of the Royal Society $B, \quad 374: 1-10$. (http://dx.doi.org/10.1098/ rstb.2017.0387)

Siddig, A. A., Ellison, A. M., Ochs, A., Villar-Leeman, C., \& M. K. Lau, 2016. How do ecologists select and use indicator species to monitor ecological change? Insights from 14 years of publication in Ecological Indicators. Ecological Indicators, 60:223-230.

Tumaneng-Diete, T., I.S. Ferguson, \& D. MacLaren, 2005. Log export restrictions and trade policies in the Philippines: bane or blessing to sustainable forest management? Forest Policy and Economics, 7:187-198.

Walpole, P., 2010. Figuring the forest figures: Understanding forest cover data in the Philippines and where we might be proceeding. Environmental Science for Social Change, http://download.essc.org.ph/forest/ESSCPWalpole_Figuring forest figures_reduded_.pdf.

Welton, L.J., C. D. Siler, A. C. Diesmos, M.L.L. Diesmos, R. Lagat, R. Causaren, \& R.M. Brown, 2012. Genetic identity, geographic range, and major distribution records for frugivorous monitor lizards of Luzon Island, Philippines. Herpetological Review, 43:226-230.

Wild Bird Club of the Philippines: Checklist of Birds of the Philippines, 2018. Available at http://www.birdwatch.ph/ downloads/WBCP-checklist-2018.xlsx

Wildlife Conservation Society of the Philippines. 1997. Philippine Red Data Book. Wildlife Conservation Society of the Philippines and Bookmark, Makati City, Philippines. $262 \mathrm{pp}$

Williams, C. B. 1964. Patterns in the balance of nature. London: Academic Press

Woodcock, P., D. P. Edwards, T. M. Fayle, R. J. Newton, C. V. Khen, S. H. Bottrell, \& K. C. Hamer, 2011. The conservation value of South East Asia's highly degraded forests: evidence from leaf-litter ants. Philosophical Transactions of the Royal Society B: Biological Sciences, 366:3256-3264.

Wu, J.G., 2013. Key concepts and research topics in landscape ecology revisited: 30 years after the Allerton Park workshop. Landscape Ecology, 28:1-11.

Yom-Tov, Y., \& E. Geffen, 2010. Recent spatial and temporal changes in body size of terrestrial vertebrates: probable causes and pitfalls. Biological Reviews, 86: 531 - 541. (doi:10.1111/j.1469-185X.2010. 00168.x) 